\title{
Soft gravitational radiation from multi-body collisions
}

\author{
Andrea Addazi ${ }^{a, b}$ and Kaiqiang Alan Zeng ${ }^{a}$ \\ ${ }^{a}$ Center for Theoretical Physics, College of Physics, Sichuan University, \\ 610065 Chengdu, China \\ ${ }^{b}$ Laboratori Nazionali di Frascati INFN, \\ Via Enrico Fermi 54, I-00044 Frascati (Roma), Italy \\ E-mail: addazi@scu.edu.cn, kaiqiangalanzeng@hotmail.com
}

ABstRACT: We derive a universal expression for the gravitational radiation energy spectrum $d E^{G W} / d \omega$ at sub-leading order emitted from a generic gravitational hard scattering of multi-particles or multi-bodies. Our result includes all $\mathcal{O}(\omega)$ corrections to the gravitational radiation flux from a generic $2 \rightarrow N$ collision, in both the cases of massless and massive particles/bodies. We also show the dependence of the radiation energy flux by the quantum spin in case of particle collisions. Then, we consider the specific case of a gravitational elastic scattering of two massive bodies, i.e. $m+M \rightarrow m+M$ with $m, M$ the masses of the two bodies respectively. We demonstrate that in this case all $\mathcal{O}(\omega)$ contributions to the energy flux exactly cancel each others. Nevertheless, we also show that, for a $2 \rightarrow 2$ inelastic scattering, the inclusion of sub-leading soft gravitons leads to a not zero radiation flux, having a simple expression in certain asymptotic regimes. Our results can be applied to the case of Black Hole collisions with possible testable implications in gravitational waves physics.

Keywords: Black Holes, Classical Theories of Gravity, Models of Quantum Gravity

ARXIV EPRINT: 2110.01194 


\section{Contents}

1 Introduction 1

2 Soft gravitational radiation from leading order: short review 3

3 Soft gravitational radiation from sub-leading order 5

3.1 Massless case: a review of the ABV result 5

$\begin{array}{lll}3.2 & \text { Massive case: a generalization of ABV result } & 7\end{array}$

4 Test in two-body elastic scattering $\quad 9$

5 Gravitational elastic scattering $\quad 10$

6 Inelastic scattering $\quad 12$

7 Conclusions and remarks $\quad 13$

$\begin{array}{ll}\text { A Gravitational elastic scattering I } & 14\end{array}$

$\begin{array}{ll}\text { B Gravitational elastic scattering II } & 16\end{array}$

$\begin{array}{ll}\text { C } \frac{d B_{1}^{(i, j)}}{d \omega} \text { for the inelastic case } & 18\end{array}$

\section{Introduction}

As it is well known, the gravitational bremsstrahlung radiation, emitted from a generic gravitational scattering, has a simple general expression in the leading-order (LO) zerofrequency limit (ZFL) derived many decades ago [1-8]. Nevertheless in the recent years the interest to soft theorems has come back with several new results about sub- and subsub-leading terms beyond the soft leading expression [9-16]. Indeed, many recent works were dedicated on several possible preservation and violation cases of universality (of soft theorems) [17-26] as well as to the connection of soft theorems with the Bondi-Metzner-Van der Burg-Sachs (BMS) symmetry group of asymptotically-flat space-time metric [27-32].

Certainly, nowadays these theoretical issues, that may appear in Weinberg's paper as academic, are now revitalized by the direct observations of Gravitational Waves from Black Hole (BH) and Neutron star mergings [33]. Indeed recently it was suggested that the gravitational memory effect related to soft theorems and BMS may be tested from future gravitational waves experiments [34]. On the other hand, the analysis of gravitational radiation in soft regime can also be important for the detection of quantum gravity effects 
such possible $\alpha^{\prime}$ corrections on radiation energy flux predicted by string theory in $\mathrm{BH}$ mergings dubbed string memory effect [35-38].

For these reasons, it is interesting to explore the possibility of using new tools developed from soft theorems and scattering amplitudes to extend the $d E^{G W} / d \omega$ results beyond the leading ZFL order, i.e. to the Next-to-leading order (NLO). Recent progresses of this program have been shown with different approaches, but substantial agreements of results [39-43].

On the other hand, ZFL radiation analysis can be compared with other results from the prospective of bremsstrahlung emission in high energy gravitational scatterings. In particular, a old standing program still in progress explores aspects of gravitational scattering at the transplanckian energy limit [44-54] (e.g. see also [55-57] for recent updates in the subject). This can lead to important insights on the information paradox in energy regimes where the $\mathrm{BH}$ should be generated [58-61] and should evaporate in form of Hawking radiation [62]. On the other hand, this can also scrutinize string theory predictions from finite size of scattering vertices and tidal excitations $[44,47,63]$ as well as possible modifications of gravity at short-distance and generalized uncertainty principle (GUP) [64, 65].

Let us consider a generic process from IN $\rightarrow$ OUT, including virtual and real soft graviton effects. Let us denote the S-matrix of such a process as ${ }^{1}$

$$
\mathcal{S}^{(0)} \rightarrow \mathcal{S}=\exp \left(\frac{d^{3} q}{\sqrt{2 \omega}}\left(\lambda_{q}^{*} a_{q}^{\dagger}-\lambda_{q} a_{q}\right)\right) \mathcal{S}^{(0)},
$$

where $a_{q}, a_{q}^{\dagger}$ are destruction and creation operators for soft gravitons of momenta $q$ and maximal cutoff energy $\Lambda \ll E$ - with $E$ the characteristic energy scale of the collision process - and $\lambda_{q}$ is a process-dependent function of $q$. Such an exponential operator applied on the bare S-matrix $\mathcal{S}^{0}$ corresponds to a coherent state operator and soft gravitons are in a coherent vacuum state.

The energy spectrum of soft graviton emitted by a generic IN $\rightarrow$ OUT process is related to the eq. (1.1) as follows

$$
{\frac{d E}{d^{3} q}}^{G W}=\frac{\hbar}{2}\left|\lambda_{q}\right|^{2}
$$

where $\lambda_{q}$ depends from the specific $\mathbf{I N} \rightarrow$ OUT scattering.

In general such an expression can be expanded in powers of $q$ and, integrating on the momenta solid angle, one can obtain a $d E^{G W} / d \omega$ with a leading term going in the ZFL $\omega \rightarrow 0$ as $d E_{0}^{G W} / d \omega \sim \omega^{-2}$.

Nevertheless, a universal factorized expression for the sub-leading graviton emission is not known and it takes the form of a differential operator acting on the bare amplitudes. Indeed the subleading soft current depends by the total angular momentum operators acting on the bare S-matrix.

The main purpose of this paper is to compute the sub-leading order of the $d E^{G W} / d \omega$ spectrum. A first step towards it was done by Bianchi, Veneziano and one of the author of

\footnotetext{
${ }^{1}$ Let us also remark that in our paper we will focus on the $3+1$ space-time dimensional case.
} 
this paper [41], considering high energy ultra-relativistic scattering of spin-less particles. Our aim is to extend the previous Addazi-Bianchi-Veneziano (ABV) analysis to the case of generic scatterings of massive particles and bodies, including non-relativistic regimes and having in mind the possible application of it for soft GW emission from $\mathrm{BH}$ mergings.

As a warm up let us consider the first three leading orders of soft graviton emissions with universal behavior

$$
\begin{aligned}
\mathcal{M}_{N+1}\left(p_{i} ; q\right) & =\sqrt{8 \pi G} \sum_{i=1}^{N}\left[\frac{p_{i} h p_{i}}{q p_{i}}+\frac{p_{i} h J_{i} q}{q p_{i}}-\frac{q J_{i} h J_{i} q}{2 q p_{i}}\right] \mathcal{M}_{N}\left(p_{i}\right)+\mathcal{O}\left(q^{2}\right) \\
& \equiv\left(S_{0}+S_{1}+S_{2}\right) \mathcal{M}_{N}\left(p_{i}\right)
\end{aligned}
$$

where $\mathcal{M}_{N+1} \equiv \mathcal{M}_{N+1}\left(p_{i} ; q\right)$ denotes a generic $(N+1)$-particle on-shell scattering amplitude including an external graviton with momentum $q^{\mu}$ and polarization $h_{\mu \nu}$.

The $q^{\mu}$ and $h_{\mu \nu}$, defined above, satisfy

$$
q^{2}=0, \quad h_{\mu \nu}=h_{\nu \mu}, \quad q^{\mu} h_{\mu \nu}=0 .
$$

The leading, subleading and sub-subleading soft factors are given by

$$
S_{0} \equiv \sqrt{8 \pi G} \sum_{i=1}^{N} \frac{p_{i} h p_{i}}{q p_{i}}, \quad S_{1}=\sqrt{8 \pi G} \sum_{i=1}^{N} \frac{p_{i} h J_{i} q}{q p_{i}}, \quad S_{2}=-\sqrt{8 \pi G} \sum_{i=1}^{N} \frac{q J_{i} h J_{i} q}{2 q p_{i}}
$$

and $J_{i}^{\mu \nu} \equiv p_{i}^{\mu} \partial_{i}^{\nu}-p_{i}^{\nu} \partial_{i}^{\mu}+S_{i}^{\mu \nu}$ denotes the total angular momentum of the $i$-th 'hard' particle.

The expression eq. (1.3) is gauge invariant under the rank-2 tensor shift

$$
h_{\mu \nu} \rightarrow h_{\mu \nu}+q_{\mu} \zeta_{\nu}+q_{\nu} \zeta_{\mu}
$$

following from the conservation of momentum and angular momentum.

The three soft terms can be related by the respective first three terms of the qexpansion of $\lambda_{q}$. This allows to compute the gravitational energy spectrum $d E^{G W} / d \omega$ after the integration over the emission direction. As we will see, such an operation involves not-trivial vector and tensor integrals that we perform in full generality.

The plan of the paper is the following. In section 2 we will briefly review Weinberg's derivation of the leading $B$-factor and $d E^{G W} / d \omega$. In section 3, we will show a complete computation of the sub-leading emission of soft radiation, in the case of massive particle collisions, after a review of the previous ABV result. In section $4 \& 5$, we will show a new surprising result: in case of $2 \rightarrow 2$ elastic scattering all sub-leading effects exactly vanish for generic particle/body collision. In section 6 , we analyze the case of $2 \rightarrow 2$ inelastic collision: we find an exact non-zero result which is, in full generality, complicated, but it has simpler expressions in certain kinematic regimes.

\section{Soft gravitational radiation from leading order: short review}

The soft theorem up to leading order is universal and the dominant behavior reads

$$
\left|\mathcal{M}_{N+1}\left(p_{i} ; q\right)\right|^{2}=8 \pi G \sum_{s= \pm 2}\left|\sum_{i=1}^{N} \frac{p_{i} h^{s} p_{i}}{q p_{i}}\right|^{2}\left|\mathcal{M}_{N}\left(p_{i}\right)\right|^{2}
$$


where notation is defined in the introduction above. The polarization tensor $h^{s}$ satisfies $h_{\mu \nu}^{-s}=\left(h_{\mu \nu}^{s}\right)^{*}$ and after tedious but straightforward manipulations one can obtain that the sum on the spins of the $h h 4$-tensor corresponds to

$$
\sum_{s= \pm 2} h_{\mu \nu}^{s} h_{\rho \sigma}^{-s}=\Pi_{\mu \nu, \rho \sigma}=\frac{1}{2}\left(\pi_{\mu \rho} \pi_{\nu \sigma}+\pi_{\mu \sigma} \pi_{\nu \rho}-\pi_{\mu \nu} \pi_{\rho \sigma}\right)
$$

where

$$
\pi_{\mu \nu}=\eta_{\mu \nu}-q_{\mu} \bar{q}_{\nu}-q_{\nu} \bar{q}_{\mu}, \quad \bar{q}^{2}=0 \quad \text { and } \quad \bar{q} q=1 .
$$

Weinberg's B-factor can be obtained after the integration over the momentum of the soft graviton in the final state $q=|q|(-1, \vec{n})$ :

$$
B_{0}=\int \frac{d^{3} q}{2|q|(2 \pi)^{3}}\left|\mathcal{M}_{N+1}\right|^{2}
$$

which corresponds to the number density as follows

$$
\frac{d N_{0}}{d \omega}=\frac{d B_{0}}{d \omega}=\int \frac{d^{3} q \delta(|q|-\omega)}{2|q|(2 \pi)^{3}}\left|\mathcal{M}_{N+1}\right|^{2}
$$

and the energy spectrum of the gravitational wave is then

$$
\frac{d E_{0}^{\mathrm{GW}}}{d \omega}=\hbar \omega \frac{d N_{0}}{d \omega}
$$

To evaluate the soft factor in eq. (2.1), one first needs to compute the polarization sum and the result reads as

$$
\sum_{i, j} \frac{p_{i}^{\mu} p_{i}^{\nu}}{q p_{i}} \Pi_{\mu \nu, \rho \sigma} \frac{p_{j}^{\rho} p_{j}^{\sigma}}{q p_{j}}=\sum_{i, j} \frac{\left(p_{i} p_{j}\right)^{2}-\frac{1}{2} p_{i}^{2} p_{j}^{2}}{q p_{i} q p_{j}}=\sum_{i, j} \frac{\left(p_{i} p_{j}\right)^{2}-\frac{1}{2} m_{i}^{2} m_{j}^{2}}{q p_{i} q p_{j}} .
$$

The evaluation of the integral in the $B$-factor

$$
I=\int \frac{d^{3} q}{|q| q p_{i} q p_{j}}
$$

leads to the well know result

$$
I=-\ln \frac{\Lambda}{\lambda}\left(\frac{2 \pi}{\beta_{i j} p_{i} p_{j}}\right) \ln \frac{1+\beta_{i j}}{1-\beta_{i j}},
$$

where we have used $\lambda$ and $\Lambda$ to denote the IR cutoff scale and the upper limit for the validity of the leading soft behavior respectively. Finally, one obtains the well-known Weinberg B-factor [2, 41, 66]:

$$
\begin{aligned}
B_{0} & =-\frac{8 \pi G}{2(2 \pi)^{3}} \sum_{i, j} \frac{\left(p_{i} p_{j}\right)^{2}-\frac{1}{2} m_{i}^{2} m_{j}^{2}}{E_{i} E_{j}} \ln \frac{\Lambda}{\lambda} \frac{2 \pi E_{i} E_{j}}{\beta_{i j} p_{i} p_{j}} \ln \frac{1+\beta_{i j}}{1-\beta_{i j}} \\
& =-\frac{G}{2 \pi} \ln \frac{\Lambda}{\lambda} \sum_{i, j} \frac{\left(p_{i} p_{j}\right)\left(1+\beta_{i j}^{2}\right)}{\beta_{i j}} \ln \frac{1+\beta_{i j}}{1-\beta_{i j}} .
\end{aligned}
$$




\section{Soft gravitational radiation from sub-leading order}

The sub-leading contribution to the GW spectrum comes from the interference between $S_{0}$ and $S_{1}$ of eq. (1.3)

$$
B_{1}=8 \pi G \int \frac{d^{3} q}{2|q|(2 \pi)^{3}} \sum_{i, j} \sum_{s= \pm 2}\left[\frac{\left(p_{i} h^{s} p_{i}\right)\left(p_{j} h^{(-s)} J_{j} q\right)}{q p_{i} q p_{j}}+(i \leftrightarrow j)\right] .
$$

Now, contrary to Winberg's $B_{0}$, the sub-leading factor $B_{1}$ involves the presence of the angular momentum operator though as acting on the $S$-matrix.

\subsection{Massless case: a review of the ABV result}

In this section, we will review the previous $\mathrm{ABV}$ result as an introduction to the main results of our paper.

Let us consider the case in which the 'hard' particles are massless: the sum over helicities of the emitted soft gravitons is

$$
\begin{aligned}
\sum_{i, j} \frac{p_{i}^{\mu} p_{i}^{\nu} p_{j}^{\rho}\left(J_{j} q\right)^{\sigma}}{q p_{i} q p_{j}} \Pi_{\mu \nu, \rho \sigma}+(i \leftrightarrow j) & =\sum_{i, j} \frac{\left(p_{i} p_{j}\right)\left(p_{i} J_{j} q\right)}{q p_{i} q p_{j}}+(i \leftrightarrow j) \\
& =\sum_{i, j} \frac{p_{i} p_{j}}{q p_{i} q p_{j}}\left[p_{i} J_{j} q+p_{j} J_{i} q\right]
\end{aligned}
$$

Thus, $B_{1}$ factor can be rewritten in a simplified form as

$$
B_{1}=8 \pi G \int \frac{d^{3} q}{2|q|(2 \pi)^{3}} \sum_{i, j} \frac{p_{i} p_{j}}{q p_{i} q p_{j}}\left[p_{i} \vec{J}_{j}+p_{j} \overleftarrow{J}_{i}\right] q
$$

where $\overleftarrow{J}_{i}$ and $\vec{J}_{j}$ act on $\mathcal{M}_{N}$ and its complex conjugate $\mathcal{M}_{N}^{*}$ respectively.

The four-vector integral that we need to evaluate for our purposes is

$$
I_{i j}^{\mu}=\int \frac{d^{3} q}{2|q|(2 \pi)^{3}} \frac{p_{i} p_{j} q^{\mu}}{q p_{i} q p_{j}}=\int \frac{d^{4} q}{(2 \pi)^{3}} \delta_{+}\left(q^{2}\right) \frac{p_{i} p_{j} q^{\mu}}{q p_{i} q p_{j}},
$$

where we have defined $\delta_{+}\left(q^{2}\right) \equiv \delta\left(q^{2}\right) \Theta\left(-q_{0}\right)$ and we used

$$
\int_{-\infty}^{\infty} d q_{0} \delta\left(q^{2}\right) \Theta\left(-q_{0}\right)=\frac{1}{2|q|}
$$

where the Heaviside step function constrains the energy to be positive.

When one considers the angel between $\vec{q}$ and $\vec{p}_{i}$ going to zero, apparent collinear divergences appear in the integral eq. (3.4). This can be explicitly seen as $q p_{i}=|q| E_{i}(-1+$ $\left.\cos \theta_{q i}\right) \sim|q| E_{i} \theta_{q i}^{2}$ as $\theta_{q i} \rightarrow 0$ while $\sin \theta_{q i} d \theta_{q i}$ inside the phase space integral $d^{3} q$ goes like $\theta_{q i} d \theta_{q i}$, thus the $\theta_{q i}$-integral becomes $d \ln \theta_{q i}$ which is divergent for $\theta_{q i} \rightarrow 0$ as well as for $\theta_{q j}$. However, we would expect that gravity is not an interaction plagued by collinear divergences. Thus, in order to avoid these apparent divergences, ABV [41] introduced the following $i j$-sum zero-equivalent shift terms as follows

$$
I_{i j}^{\mu} \rightarrow \tilde{I}_{i j}^{\mu}=\int \frac{d^{4} q}{(2 \pi)^{3}} \delta_{+}\left(q^{2}\right) \frac{\left[\left(p_{i} p_{j}\right) q^{\mu}-\left(q p_{j}\right) p_{i}^{\mu}-\left(q p_{i}\right) p_{j}^{\mu}\right]}{\left(p_{i} q\right)\left(p_{j} q\right)},
$$


the shift terms in eq. (3.6) vanish after summing over $i$ and $j$ thanks to momentum and angular momentum conservations.

The four-vector integral eq. (3.6) can be rewritten in a Lorentz contravariant form as follows

$$
K_{i j}^{\mu}(P, \Lambda)=\int \frac{d^{4} q}{(2 \pi)^{3}} \frac{\delta_{+}\left(q^{2}\right) \delta\left(\frac{q P}{\Lambda^{2}}+1\right)}{\left(q p_{i}\right)\left(q p_{j}\right)}\left[\left(p_{i} p_{j}\right) q-\left(q p_{j}\right) p_{i}-\left(q p_{i}\right) p_{j}\right]^{\mu},
$$

where $P$ is an arbitrary four vector and $\Lambda$ is a constant with the dimension of energy.

In a generic $n \rightarrow m$ process, $P$ can be identified as the total momentum of $n$ incoming (or $m$ outgoing) particles and thus we can choose it as $P=\left(E_{\mathrm{CM}}, 0\right)$. With this choice, $\Lambda^{2}=\sqrt{s} \hbar \omega_{0}$ where $s=-P^{2}=E_{\mathrm{CM}}^{2}$ is the Mandelstam variable of the corresponding channel and $\omega_{0}$ is the center-of-mass frequency at which we wish to compute the energy spectrum. Then the Dirac-Delta can be rewritten as

$$
\delta\left(\frac{q P}{\Lambda^{2}}+1\right)=\delta\left(1-\frac{\omega}{\omega_{0}}\right) .
$$

The Lorentz-invariant (graviton number) spectrum $d B_{1} / d \omega_{0}$ is then given by

$$
\frac{d B_{1}}{d \omega_{0}}=8 \pi G \sum_{i, j} \frac{K_{i j}^{\mu}\left(P, \omega_{0}\right)}{\omega_{0}}\left[p_{i} \vec{J}_{j}+p_{j} \overleftarrow{J}_{i}\right]_{\mu}
$$

With Lorentz covariance, $K_{i j}^{\mu}$ can be expanded as

$$
K_{i j}^{\mu}\left(p_{i} p_{j}\right)=K_{P}(s) P^{\mu}+K_{i}\left(P p_{i}\right) p_{i}^{\mu}+K_{j}\left(P p_{j}\right) p_{j}^{\mu},
$$

since $K_{i j}^{\mu}$ is orthogonal to both $p_{i}$ and $p_{j}, K_{i j}^{\mu}$ can be rewritten in the form

$$
K_{i j}^{\mu}=K\left[\left(p_{i} p_{j}\right) P^{\mu}-\left(P p_{j}\right) p_{i}^{\mu}-\left(P p_{i}\right) p_{j}^{\mu}\right] \equiv K\left(p_{i} p_{j}\right) Q_{i j}^{\mu},
$$

where we have defined $K \equiv \frac{K_{P}}{p_{i} p_{j}}$ and a new vector

$$
Q_{i j}^{\mu} \equiv P^{\mu}-\frac{P p_{j}}{p_{i} p_{j}} p_{i}^{\mu}-\frac{P p_{i}}{p_{i} p_{j}} p_{j}^{\mu} .
$$

The main integrals involved in eq. (3.8) are

$$
\int \frac{d^{3} q}{|q|} \delta\left(\omega-\omega_{0}\right) \frac{\left(p_{i} p_{j}\right)(q P)}{q p_{i} q p_{j}}=-\frac{\left(p_{i} p_{j}\right) \sqrt{s}}{E_{i} E_{j}} J=\frac{2 \pi \sqrt{s}}{\beta_{i j}} \ln \frac{1+\beta_{i j}}{1-\beta_{i j}},
$$

and

$$
\begin{aligned}
\int \frac{d^{3} q}{|q|} \delta\left(\omega-\omega_{0}\right)\left(\frac{P p_{i}}{q p_{i}}+\frac{P p_{j}}{q p_{j}}\right) & =\sqrt{s}\left(L_{i}+L_{j}\right) \\
& =-2 \pi \sqrt{s}\left(\frac{1}{\left|\vec{v}_{i}\right|} \ln \frac{1-\left|\vec{v}_{i}\right|}{1+\left|\vec{v}_{i}\right|}+\frac{1}{\left|\vec{v}_{j}\right|} \ln \frac{1-\left|\vec{v}_{j}\right|}{1+\left|\vec{v}_{j}\right|}\right) .
\end{aligned}
$$


In the massless limit, the above expressions has the form

$$
\int \frac{d^{3} q}{|q|} \delta\left(\omega-\omega_{0}\right) \frac{\left(p_{i} p_{j}\right)(q P)}{q p_{i} q p_{j}}=4 \pi \sqrt{s} \ln \left(-\frac{2 p_{i} p_{j}}{m_{i} m_{j}}\right)
$$

and

$$
\int \frac{d^{3} q}{|q|} \delta\left(\omega-\omega_{0}\right)\left(\frac{P p_{i}}{q p_{i}}+\frac{P p_{j}}{q p_{j}}\right)=-4 \pi \sqrt{s} \ln \frac{m_{i} m_{j}}{4 E_{i} E_{j}} .
$$

Contracting $K_{i j}^{\mu}$ with $P^{\mu}$, we get from eq. (3.7):

$$
\begin{aligned}
K_{i j} P & =\int \frac{d^{3} q}{2|q|(2 \pi)^{3}} \delta\left(1-\frac{\omega}{\omega_{0}}\right)\left[\frac{\left(p_{i} p_{j}\right)(P q)}{\left(q p_{i}\right)\left(q p_{j}\right)}-\left(\frac{P p_{i}}{q p_{i}}+\frac{P p_{j}}{q p_{j}}\right)\right] \\
& =-\frac{4 \pi \omega_{0} \sqrt{s}}{2(2 \pi)^{3}}\left[\ln \left(-\frac{2 p_{i} p_{j}}{m_{i} m_{j}}\right)+\ln \left(\frac{m_{i} m_{j}}{4 E_{i} E_{j}}\right)\right]=-\frac{\omega_{0} \sqrt{s}}{4 \pi^{2}} \ln \left(-\frac{p_{i} p_{j}}{2 E_{i} E_{j}}\right)
\end{aligned}
$$

and from eq. (3.10):

$$
K_{i j} P=K\left[\left(p_{i} p_{j}\right) P^{2}-2\left(P p_{j}\right)\left(P p_{i}\right)\right] .
$$

$K$ is then determined by equating eq. (3.16) and eq. (3.17)

$$
K\left(p_{i} p_{j}\right)=\frac{\omega_{0} \sqrt{s}}{4 \pi^{2} \tilde{s}_{i j}} \ln \left(-\frac{p_{i} p_{j}}{2 E_{i} E_{j}}\right),
$$

where we have introduced the quantity

$$
\tilde{s}_{i j}=-Q_{i j}^{2}=s+\frac{2\left(P p_{i}\right)\left(P p_{j}\right)}{p_{i} p_{j}} .
$$

Substituting the above result into eq. (3.7) and renaming $\omega_{0}$ as $\omega$, we have a sub-leading factor as follows:

$$
\begin{aligned}
\frac{d B_{1}}{d \omega} & =-2 \frac{G \sqrt{s}}{\pi} \sum_{i, j} \frac{1}{\tilde{s}_{i j}} \ln \left(-\frac{p_{i} p_{j}}{2 E_{i} E_{j}}\right) Q_{i j}^{\mu}\left[p_{i} \vec{J}_{j}+p_{j} \overleftarrow{J}_{i}\right]_{\mu} \\
& =-2 \frac{G \sqrt{s}}{\pi} \sum_{i, j} \frac{\left(p_{i} p_{j}\right)}{\tilde{s}_{i j}} \ln \left(-\frac{p_{i} p_{j}}{2 E_{i} E_{j}}\right) Q_{i j}^{\mu}\left[\vec{\partial}_{j}+\overleftarrow{\partial}_{i}\right]_{\mu}
\end{aligned}
$$

In [41], ABV showed that the sub-leading correction $d B_{1} / d \omega$ vanishes for a $2 \rightarrow 2$ process by direct computation in the Briet frame (see figure 1). We will show in the following sections that the zero result is also re-obtained in the massive case. In next section, we will derive a new $B$-factor for the massive case.

\subsection{Massive case: a generalization of $\mathrm{ABV}$ result}

In this section, we will generalize the above discussion to the massive case.

First, we notice that the polarization sum in the massive case has an additional term and it reads as

$$
\begin{aligned}
& \sum_{i, j} \frac{p_{i}^{\mu} p_{i}^{\nu} p_{j}^{\rho}\left(J_{j} q\right)^{\sigma}}{q p_{i} q p_{j}} \Pi_{\mu \nu, \rho \sigma}+(i \leftrightarrow j) \\
= & \sum_{i, j} \frac{\left(p_{i} p_{j}\right)\left(p_{i} J_{j} q\right)-\frac{1}{2} p_{i}^{2}\left(p_{j} J_{j} q\right)}{q p_{i} q p_{j}}+(i \leftrightarrow j) \\
= & \sum_{i, j} \frac{p_{i} p_{j}}{q p_{i} q p_{j}}\left[p_{i} J_{j} q+p_{j} J_{i} q\right]-\frac{1}{2} \frac{1}{q p_{i} q p_{j}}\left[p_{i}^{2}\left(p_{j} J_{j} q\right)+p_{j}^{2}\left(p_{i} J_{i} q\right)\right] .
\end{aligned}
$$




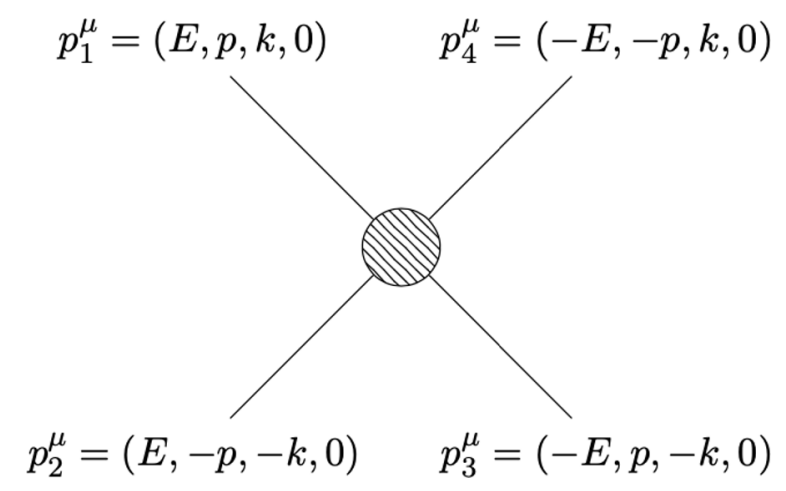

Figure 1. $2 \rightarrow 2$ scattering in Breit frame.

Considering the polarization sum in eq. (3.1) with eq. (3.21), the sub-leading $B$-factor becomes

$$
\begin{aligned}
B_{1}= & 8 \pi G \int \frac{d^{3} q}{2|q|(2 \pi)^{3}} \sum_{i, j}\left\{\frac{p_{i} p_{j} q^{\mu}}{q p_{i} q p_{j}}\left(p_{i} \vec{J}_{j}+p_{j} \overleftarrow{J}_{i}\right)_{\mu}\right. \\
& \left.-\frac{1}{2} \frac{q^{\mu}}{q p_{i} q p_{j}}\left[p_{i}^{2}\left(p_{j} \vec{J}_{j}\right)_{\mu}+p_{j}^{2}\left(p_{i} \overleftarrow{J}_{i}\right)_{\mu}\right]\right\}
\end{aligned}
$$

Then $B_{1}$ can be rewritten in terms of the integral eq. (3.4) as follows

$$
B_{1}=8 \pi G \sum_{i, j} I_{i j}^{\mu}\left\{\left(p_{i} \vec{J}_{j}+p_{j} \overleftarrow{J}_{i}\right)_{\mu}-\frac{1}{2}\left[\frac{p_{i}^{2}}{p_{i} p_{j}}\left(p_{j} \vec{J}_{j}\right)_{\mu}+\frac{p_{j}^{2}}{p_{i} p_{j}}\left(p_{i} \overleftarrow{J}_{i}\right)_{\mu}\right]\right\}
$$

Following the same procedure in section 3.1, one gets the number density spectrum

$$
\frac{d B_{1}}{d \omega_{0}}=-8 \pi G \sum_{i, j} \frac{K_{i j}^{\mu}\left(P, \omega_{0}\right)}{\omega_{0}}\left[p_{i} \vec{J}_{j}-\frac{1}{2} \frac{p_{i}^{2}}{p_{i} p_{j}}\left(p_{j} \vec{J}_{j}\right)+(i \leftrightarrow j)\right]
$$

where $K_{i j}^{\mu}$ is given by

$$
K_{i j}^{\mu}\left(P, \omega_{0}\right)=\int \frac{d^{4} q}{(2 \pi)^{3}} \frac{\delta_{+}\left(q^{2}\right) \delta\left(1-\frac{\omega}{\omega_{0}}\right)}{\left(q p_{i}\right)\left(q p_{j}\right)}\left[\left(p_{i} p_{j}\right) q-\left(q p_{j}\right) p_{i}-\left(q p_{i}\right) p_{j}\right]^{\mu},
$$

and it can be written in the form

$$
K_{i j}^{\mu}=K\left[\left(p_{i} p_{j}\right) P^{\mu}-\left(P p_{j}\right) p_{i}^{\mu}-\left(P p_{i}\right) p_{j}^{\mu}\right] \equiv K\left(p_{i} p_{j}\right) Q_{i j}^{\mu} .
$$

Contracting $K_{i j}^{\mu}$ with $P_{\mu}$, with eq. (3.12) and eq. (3.14) and from eq. (3.25), one obtains

$$
\begin{aligned}
K_{i j} P & =\int \frac{d^{3} q}{2|q|(2 \pi)^{3}} \delta\left(1-\frac{\omega}{\omega_{0}}\right)\left[\frac{\left(p_{i} p_{j}\right)(q P)}{q p_{i} q p_{j}}-\left(\frac{P p_{i}}{q p_{i}}+\frac{P p_{j}}{q p_{j}}\right)\right] \\
& =-\frac{\omega_{0}}{2(2 \pi)^{3}}\left[\frac{2 \pi \sqrt{s}}{\beta_{i j}} \ln \frac{1+\beta_{i j}}{1-\beta_{i j}}+2 \pi \sqrt{s}\left(\frac{1}{\left|\vec{v}_{i}\right|} \ln \frac{1-\left|\vec{v}_{i}\right|}{1+\left|\vec{v}_{i}\right|}+\frac{1}{\left|\vec{v}_{j}\right|} \ln \frac{1-\left|\vec{v}_{j}\right|}{1+\left|\vec{v}_{j}\right|}\right)\right],
\end{aligned}
$$

and from eq. (3.26)

$$
K_{i j} P=K\left[\left(p_{i} p_{j}\right) P^{2}-2\left(P p_{j}\right)\left(P p_{i}\right)\right]
$$


Thus $K$ is determined by

$$
K\left(p_{i} p_{j}\right)=\frac{\omega_{0} \sqrt{s}}{8 \pi^{2} \tilde{s}_{i j}}\left[\frac{1}{\beta_{i j}} \ln \frac{1+\beta_{i j}}{1-\beta_{i j}}+\left(\frac{1}{\left|\vec{v}_{i}\right|} \ln \frac{1-\left|\vec{v}_{i}\right|}{1+\left|\vec{v}_{i}\right|}+\frac{1}{\left|\vec{v}_{j}\right|} \ln \frac{1-\left|\vec{v}_{j}\right|}{1+\left|\vec{v}_{j}\right|}\right)\right] .
$$

Substituting the above $K$-factor into eq. (3.24) gives

$$
\begin{aligned}
\frac{d B_{1}}{d \omega}= & -\frac{G \sqrt{s}}{\pi} \sum_{i, j} \frac{1}{\tilde{s}_{i j}}\left[\frac{1}{\beta_{i j}} \ln \frac{1+\beta_{i j}}{1-\beta_{i j}}+\left(\frac{1}{\left|\vec{v}_{i}\right|} \ln \frac{1-\left|\vec{v}_{i}\right|}{1+\left|\vec{v}_{i}\right|}+\frac{1}{\left|\vec{v}_{j}\right|} \ln \frac{1-\left|\vec{v}_{j}\right|}{1+\left|\vec{v}_{j}\right|}\right)\right] \\
& \times Q_{i j}^{\mu}\left[p_{i} \vec{J}_{j}+p_{j} \overleftarrow{J}_{i}\right]_{\mu} .
\end{aligned}
$$

Considering that

$$
Q_{i j}^{\mu}\left[p_{i} \vec{J}_{j}+p_{j} \overleftarrow{J}_{i}\right]_{\mu}=\left(p_{i} p_{j}\right) Q_{i j}^{\mu}\left(\overleftarrow{\partial}_{i}+\vec{\partial}_{j}\right)_{\mu}+\frac{P p_{i}}{p_{i} p_{j}} p_{j}^{2}\left(\vec{\partial}_{j} p_{i}\right)+\frac{P p_{j}}{p_{i} p_{j}} p_{i}^{2}\left(\overleftarrow{\partial}_{i} p_{j}\right)
$$

we finally obtain the new general subleading $B$-factor for the massive case

$$
\begin{aligned}
\frac{d B_{1}}{d \omega}= & -\frac{G \sqrt{s}}{\pi} \sum_{i, j} \frac{1}{\tilde{s}_{i j}}\left[\frac{1}{\beta_{i j}} \ln \frac{1+\beta_{i j}}{1-\beta_{i j}}+\left(\frac{1}{\left|\vec{v}_{i}\right|} \ln \frac{1-\left|\vec{v}_{i}\right|}{1+\left|\vec{v}_{i}\right|}+\frac{1}{\left|\vec{v}_{j}\right|} \ln \frac{1-\left|\vec{v}_{j}\right|}{1+\left|\vec{v}_{j}\right|}\right)\right] \\
& \times\left[\left(p_{i} p_{j}\right) Q_{i j}^{\mu}\left(\overleftarrow{\partial}_{i}+\vec{\partial}_{j}\right)_{\mu}+\frac{P p_{i}}{p_{i} p_{j}} p_{j}^{2}\left(\vec{\partial}_{j} p_{i}\right)+\frac{P p_{j}}{p_{i} p_{j}} p_{i}^{2}\left(\overleftarrow{\partial}_{i} p_{j}\right)\right]
\end{aligned}
$$

\section{Test in two-body elastic scattering}

In this section, we use the result (3.29) to study a two-body elastic collision. The kinematics for this process is similar to the one shown in figure 1 with the difference that here we consider all the 'hard' particles to be massive and with the same mass $m$. Thus the energy-momentum conservation condition becomes $E^{2}=p^{2}+k^{2}+m^{2}$ and the Mandelstam variables satisfy the relation: $s+t+u=4 m^{2}$. The contribution from the $(i, j)$-pair takes the form

$$
\begin{aligned}
\frac{d B_{1}^{(i, j)}}{d \omega}= & \eta_{i} \eta_{j} \frac{G}{\pi} \frac{1-\vec{v}_{i} \cdot \vec{v}_{j}}{1+\vec{v}_{i} \cdot \vec{v}_{j}}\left[\frac{1}{\beta_{i j}} \ln \frac{1+\beta_{i j}}{1-\beta_{i j}}+\frac{2 E}{\sqrt{p^{2}+k^{2}}} \ln \frac{E-\sqrt{p^{2}+k^{2}}}{E+\sqrt{p^{2}+k^{2}}}\right] \\
& \times\left\{E^{2}\left[\delta_{0}^{\mu}\left(1+\vec{v}_{i} \cdot \vec{v}_{j}\right)+\delta_{r}^{\mu}\left(\vec{v}_{i}+\vec{v}_{j}\right)^{r}\right]\left(\overleftarrow{\partial}_{i}+\vec{\partial}_{j}\right)_{\mu}\right. \\
& \left.-\frac{m^{2}}{1-\vec{v}_{i} \cdot \vec{v}_{j}}\left[\left(\delta_{0}^{\mu}+\delta_{r}^{\mu}\left(\vec{v}_{i}\right)^{r}\right)\left(\vec{\partial}_{j}\right)_{\mu}+\left(\delta_{0}^{\mu}+\delta_{r}^{\mu}\left(\vec{v}_{j}\right)^{r}\right)\left(\overleftarrow{\partial}_{i}\right)_{\mu}\right]\right\},
\end{aligned}
$$

where $\beta_{i j}$ and $\left|\vec{v}_{i}\right|$ are given by

$$
\left|\vec{v}_{i}\right|=\sqrt{\frac{p^{2}+k^{2}}{E^{2}}} \text { and } \beta_{i j} \equiv \sqrt{1-\frac{m^{4}}{\left(p_{i} \cdot p_{j}\right)^{2}}} .
$$


First considering the case $i, j=1,2$ and $i, j=3,4$, a direct computation gives

$$
\begin{aligned}
& \frac{d B_{1}^{(1,2)}}{d \omega}+\frac{d B_{1}^{(2,1)}}{d \omega} \\
= & \frac{2 G}{\pi}\left[\frac{E^{2}+p^{2}+k^{2}}{2 E \sqrt{p^{2}+k^{2}}} \ln \frac{E^{2}+p^{2}+k^{2}+2 E \sqrt{p^{2}+k^{2}}}{E^{2}+p^{2}+k^{2}-2 E \sqrt{p^{2}+k^{2}}}+\frac{2 E}{\sqrt{p^{2}+k^{2}}} \ln \frac{E-\sqrt{p^{2}+k^{2}}}{E+\sqrt{p^{2}+k^{2}}}\right] \\
& \times\left\{\left(p^{2}+k^{2}\right) \overleftrightarrow{\partial}_{E}+E\left(p \overleftrightarrow{\partial}_{p}+k \overleftrightarrow{\partial}_{k}\right)\right\},
\end{aligned}
$$

and

$$
\begin{aligned}
& \frac{d B_{1}^{(3,4)}}{d \omega}+\frac{d B_{1}^{(4,3)}}{d \omega} \\
= & -\frac{2 G}{\pi}\left[\frac{E^{2}+p^{2}+k^{2}}{2 E \sqrt{p^{2}+k^{2}}} \ln \frac{E^{2}+p^{2}+k^{2}+2 E \sqrt{p^{2}+k^{2}}}{E^{2}+p^{2}+k^{2}-2 E \sqrt{p^{2}+k^{2}}}+\frac{2 E}{\sqrt{p^{2}+k^{2}}} \ln \frac{E-\sqrt{p^{2}+k^{2}}}{E+\sqrt{p^{2}+k^{2}}}\right] \\
& \times\left\{\left(p^{2}+k^{2}\right) \overleftrightarrow{\partial}_{E}+E\left(p \overleftrightarrow{\partial}_{p}+k \overleftrightarrow{\partial}_{k}\right)\right\} .
\end{aligned}
$$

Thus, we obtain

$$
\frac{d B_{1}^{(1,2)}}{d \omega}+\frac{d B_{1}^{(2,1)}}{d \omega}+\frac{d B_{1}^{(3,4)}}{d \omega}+\frac{d B_{1}^{(4,3)}}{d \omega}=0
$$

which means that the contributions from $(1,2)$ and $(3,4)$ cancel with each other.

For $i, j=1,3$, after a long but straightforward computation, one finds

$$
\begin{aligned}
\frac{d B_{1}^{(1,3)}}{d \omega}= & -\frac{G}{\pi} \frac{E^{2}+p^{2}-k^{2}}{E^{2}-p^{2}+k^{2}}\left[\frac{E^{2}+p^{2}-k^{2}}{2 p \sqrt{E^{2}-k^{2}}} \ln \frac{E^{2}+p^{2}-k^{2}+2 p \sqrt{E^{2}-k^{2}}}{E^{2}+p^{2}-k^{2}-2 p \sqrt{E^{2}-k^{2}}}\right. \\
& \left.+\frac{2 E}{\sqrt{p^{2}+k^{2}}} \ln \frac{E-\sqrt{p^{2}+k^{2}}}{E+\sqrt{p^{2}+k^{2}}}\right]\left\{E^{2}\left[\frac{E^{2}-p^{2}+k^{2}}{E^{2}}\left(\overleftarrow{\partial}_{E}-\vec{\partial}_{E}\right)+\frac{2 k}{E}\left(\overleftarrow{\partial}_{k}-\vec{\partial}_{k}\right)\right]\right. \\
& \left.-\frac{E^{2} m^{2}}{E^{2}+p^{2}-k^{2}}\left[\left(-\vec{\partial}_{E}+\overleftarrow{\partial}_{E}\right)+\frac{p}{E}\left(\vec{\partial}_{p}-\overleftarrow{\partial}_{p}\right)+\frac{k}{E}\left(-\vec{\partial}_{k}+\overleftarrow{\partial}_{k}\right)\right]\right\} \\
= & -\frac{d B_{1}^{(3,1)}}{d \omega}
\end{aligned}
$$

Once again, the total contribution from $(1,3)$-pair vanishes. We have also checked that the same happens for the other pairs: $(1,4),(2,3),(2,4)$, proving that $d B_{1} / d \omega=0$ is also valid in the case of massive two-body collisions with all particles having the same mass.

\section{$5 \quad$ Gravitational elastic scattering}

In this section, we apply the new sub-leading differential $B$-factor obtained in previous sections to the case of a gravitational elastic scattering.

Indeed, from a classical prospective this can correspond to the case of a test light body elastically deflected but not captured by a Black Hole of mass $M$. 


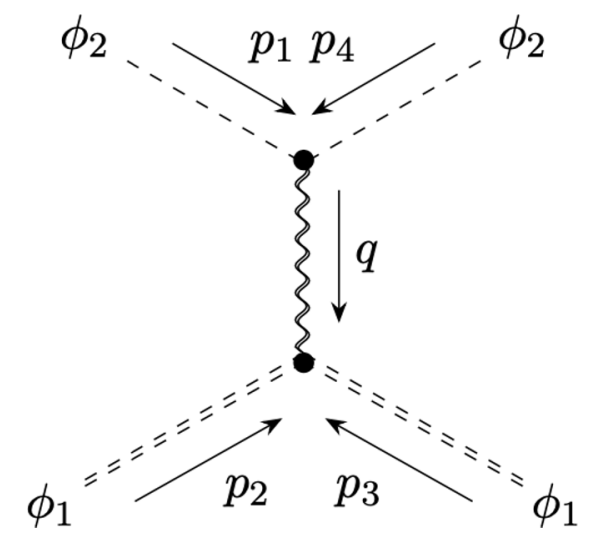

Figure 2. Feynman's diagram of gravitational elastic scattering $\phi_{1}\left(p_{1}\right) \phi_{2}\left(p_{2}\right) \rightarrow \phi_{1}\left(p_{3}\right) \phi_{2}\left(p_{4}\right)$, where $\phi_{1,2}$ are two scalar fields (dashed lines), the curly line represent the propagator of the graviton field and $p_{1,2,3,4}$ are the four-momenta of in and out states respectively.

For the case in which the test body has a mass $m \ll M$, the differential $B$-factor has an asymptotic expression in the limit of $m \rightarrow 0$ (see appendix A for detail derivation) as follows

$$
\frac{d B_{1}}{d \omega} \sim \frac{G M}{\pi}\left(6+\frac{2}{3} \frac{P^{2}}{M^{2}}\right)\left(\vec{p}_{f} \overleftrightarrow{\partial}_{\vec{p}_{f}}-\vec{p}_{i} \overleftrightarrow{\partial}_{\vec{p}_{i}}\right)
$$

where $P=|\vec{p}|, M$ is the mass of the black hole, $\vec{p}_{i}, \vec{p}_{f}$ are initial and final momenta in the CM frame. Then the soft sub-leading radiation in ZFL can be obtained applying the differential B-factor in eq. (5.1) on the amplitude of gravitational $2 \rightarrow 2$ elastic scattering

$$
\mathcal{M}^{*} \frac{d B_{1}}{d \omega} \mathcal{M}
$$

where the partial derivatives with left (right) arrow act on $\mathcal{M}^{*}(\mathcal{M})$.

From field theory prospective, let us start from the Einstein-Hilbert action coupled to two scalar fields $\phi_{1}$ and $\phi_{2}$

$$
\mathcal{S}=\int d^{4} x \sqrt{-g}\left[\frac{1}{16 \pi G} R-\frac{1}{2} \partial^{\mu} \phi_{1} \partial_{\mu} \phi_{1}-\frac{1}{2} M^{2} \phi_{1}^{2}-\frac{1}{2} \partial^{\nu} \phi_{2} \partial_{\nu} \phi_{2}-\frac{1}{2} m^{2} \phi_{2}^{2}\right] .
$$

Indeed, for the moment, as an illustrative example, we consider spin-less field gravitational scatterings (see figure 2). The amplitude for this classical tree-level diagram is $[67]$

$$
\mathcal{M}=\frac{16 \pi G}{q^{2}}\left(M^{2} m^{2}-2\left(p_{1} \cdot p_{2}\right)^{2}-\left(p_{1} \cdot p_{2}\right) q^{2}\right),
$$

where $p_{1}, p_{2}\left(p_{3}, p_{4}\right)$ are incoming (outgoing) momenta, $p_{1}^{2}=p_{4}^{2}=-m^{2}, p_{2}^{2}=p_{3}^{2}=-M^{2}$ and the momentum transfer is $q=p_{1}+p_{4}=-\left(p_{2}+p_{3}\right)$. With the kinematics discussed in appendix $\mathrm{A}$, the amplitude in the center of mass frame becomes

$$
\begin{aligned}
\mathcal{M} & =\frac{8 \pi G}{\left(P^{2}+k k^{\prime}-p p^{\prime}\right)}\left[M^{2} m^{2}-2 P\left(M+P+\frac{P^{2}}{2 M}\right)\left(P\left(M+\frac{P^{2}}{2 M}\right)-k k^{\prime}+p p^{\prime}\right)\right] \\
& \sim \frac{8 \pi G}{\left(P^{2}+k k^{\prime}-p p^{\prime}\right)}\left[M^{2} m^{2}-2 P(M+P)\left(M P-k k^{\prime}+p p^{\prime}\right)\right] .
\end{aligned}
$$


The derivatives of this amplitude give

$$
\begin{gathered}
\vec{\partial}_{p} \mathcal{M}=\frac{8 \pi G}{\left(P^{2}+k k^{\prime}-p p^{\prime}\right)^{2}} p^{\prime}\left(M^{2} m^{2}-2 P^{2}(M+P)^{2}\right)=\mathcal{M}^{*} \overleftarrow{\partial}_{p}, \\
\vec{\partial}_{k} \mathcal{M}=\frac{8 \pi G}{\left(P^{2}+k k^{\prime}-p p^{\prime}\right)^{2}} k^{\prime}\left(2 P^{2}(M+P)^{2}-M^{2} m^{2}\right)=\mathcal{M}^{*} \overleftarrow{\partial}_{k}, \\
\vec{\partial}_{p^{\prime}} \mathcal{M}=\frac{8 \pi G}{\left(P^{2}+k k^{\prime}-p p^{\prime}\right)^{2}} p\left(M^{2} m^{2}-2 P^{2}(M+P)^{2}\right)=\mathcal{M}^{*} \overleftarrow{\partial}_{p^{\prime}}, \\
\vec{\partial}_{k^{\prime}} \mathcal{M}=\frac{8 \pi G}{\left(P^{2}+k k^{\prime}-p p^{\prime}\right)^{2}} k\left(2 P^{2}(M+P)^{2}-M^{2} m^{2}\right)=\mathcal{M}^{*} \overleftarrow{\partial}_{k^{\prime}} .
\end{gathered}
$$

Then it is easy to check that

$$
\begin{aligned}
& p\left[\left(\mathcal{M}^{*} \overleftarrow{\partial}_{p}\right) \mathcal{M}+\mathcal{M}^{*}\left(\vec{\partial}_{p} \mathcal{M}\right)\right]-p^{\prime}\left[\left(\mathcal{M}^{*} \overleftarrow{\partial}_{p^{\prime}}\right) \mathcal{M}+\mathcal{M}^{*}\left(\vec{\partial}_{p^{\prime}} \mathcal{M}\right)\right]=0 \\
& k\left[\left(\mathcal{M}^{*} \overleftarrow{\partial}_{k}\right) \mathcal{M}+\mathcal{M}^{*}\left(\vec{\partial}_{k} \mathcal{M}\right)\right]-k^{\prime}\left[\left(\mathcal{M}^{*} \overleftarrow{\partial}_{k^{\prime}}\right) \mathcal{M}+\mathcal{M}^{*}\left(\vec{\partial}_{k^{\prime}} \mathcal{M}\right)\right]=0
\end{aligned}
$$

which implies

$$
\mathcal{M}\left(p^{\prime} \overleftrightarrow{\partial}_{p^{\prime}}+k^{\prime} \overleftrightarrow{\partial}_{k^{\prime}}-p \overleftrightarrow{\partial}_{p}-k \overleftrightarrow{\partial}_{k}\right) \mathcal{M}^{*}=0
$$

Thus once again, we get a vanishing result

$$
\mathcal{M}^{*} \frac{d B_{1}}{d \omega} \mathcal{M}=0
$$

even if the differential $B$-factor is non-zero. Furthermore, in appendix B, we find that eq. (5.8) is still re-obtained in the case of massive $m$ test particle.

\section{$6 \quad$ Inelastic scattering}

Here, we will show that $d B_{1} / d \omega$ gives a finite non-zero result in the $2 \rightarrow 2$ inelastic scattering case. Indeed, in the case of test particle deflected by a $\mathrm{BH}$, it is reasonable to consider the elastic scattering regime only for small deflection angles. In case of harder deflections we would expect that inelastic channels would be opened.

Following similar discussion as appendix A, but this time take

$$
p^{2}+k^{2}=P^{2} \neq P^{\prime 2}=p^{\prime 2}+k^{\prime 2}
$$

with eq. (3.29), we can get the contributions from different particle pairs. As shown in appendix $\mathrm{C}$, however, the result is a long expression.

Nevertheless, in the non-relativistic regime, performing a Taylor series expansion up to the second order of $O(P / M)$ and $O\left(P^{\prime} / M^{\prime}\right)$, the six contributions simplify to

$$
\begin{aligned}
& \frac{d B_{1}^{(1,2)}}{d \omega}+\frac{d B_{1}^{(2,1)}}{d \omega} \sim \frac{G M}{\pi}\left(-2+\frac{1}{3} \frac{P^{2}}{M^{2}}\right)\left(P \overleftrightarrow{\partial}_{P}+p \overleftrightarrow{\partial}_{p}+k \overleftrightarrow{\partial}_{k}\right) \\
& \frac{d B_{1}^{(3,4)}}{d \omega}+\frac{d B_{1}^{(4,3)}}{d \omega} \sim \frac{G M^{\prime}}{\pi}\left(2-\frac{1}{3} \frac{P^{\prime 2}}{M^{\prime 2}}\right)\left(P^{\prime} \overleftrightarrow{\partial}_{P^{\prime}}+p^{\prime} \overleftrightarrow{\partial}_{p^{\prime}}+k^{\prime} \overleftrightarrow{\partial}_{k^{\prime}}\right)
\end{aligned}
$$




$$
\begin{aligned}
& \frac{d B_{1}^{(2,4)}}{d \omega}+\frac{d B_{1}^{(4,2)}}{d \omega} \sim \frac{G M^{\prime}}{\pi}\left(-2+\frac{1}{3} \frac{P^{\prime 2}}{M^{\prime 2}}\right)\left(P \overleftrightarrow{\partial}_{P}+p \overleftrightarrow{\partial}_{p}+k \overleftrightarrow{\partial}_{k}\right) \\
& \frac{d B_{1}^{(1,3)}}{d \omega}+\frac{d B_{1}^{(3,1)}}{d \omega} \sim \frac{G M}{\pi}\left(-2+\frac{1}{3} \frac{P^{2}}{M^{2}}\right)\left(-P^{\prime} \overleftrightarrow{\partial}_{P^{\prime}}-p^{\prime} \overleftrightarrow{\partial}_{p^{\prime}}-k^{\prime} \overleftrightarrow{\partial}_{k^{\prime}}\right) \\
& \frac{d B_{1}^{(1,4)}}{d \omega}+\frac{d B_{1}^{(4,1)}}{d \omega} \sim \frac{G}{\pi}\left[-2-\frac{2}{3}\left(\frac{P^{2}}{M^{2}}+\frac{P^{\prime 2}}{M^{\prime 2}}\right)\right]\left(p M^{\prime} \overleftrightarrow{\partial}_{p}+k M^{\prime} \overleftrightarrow{\partial}_{k}-p^{\prime} M \overleftrightarrow{\partial}_{p^{\prime}}-k^{\prime} M \overleftrightarrow{\partial}_{k^{\prime}}\right) \\
& \frac{d B_{1}^{(2,3)}}{d \omega}+\frac{d B_{1}^{(3,2)}}{d \omega}= \frac{2 G}{\pi} \frac{P P^{\prime}-\left(p p^{\prime}-k k^{\prime}\right)}{P P^{\prime}+\left(p p^{\prime}-k k^{\prime}\right)} \ln \left[\frac{P P^{\prime}-\left(p p^{\prime}-k k^{\prime}\right)}{2 P P^{\prime}}\right]\left\{\left[P P^{\prime}+\left(p p^{\prime}-k k^{\prime}\right)\right]\right. \\
&\left.\times\left(\overleftrightarrow{\partial}_{P}-\overleftrightarrow{\partial}_{P^{\prime}}\right)+\left(p P^{\prime}+p^{\prime} P\right)\left(\overleftrightarrow{\partial}_{p}-\overleftrightarrow{\partial}_{p^{\prime}}\right)+\left(k P^{\prime}-k^{\prime} P\right)\left(\overleftrightarrow{\partial}_{k}+\overleftrightarrow{\partial}_{k^{\prime}}\right)\right\}
\end{aligned}
$$

Summing the single contributions, we find that the total sub-leading $B$-fractor for the inelastic scattering is dominated by the following leading term:

$$
\begin{aligned}
\frac{d B_{1}}{d \omega}= & {\left[\frac{G M}{\pi}\left(2-\frac{1}{3} \frac{P^{2}}{M^{2}}\right)+\frac{G M^{\prime}}{\pi}\left(2-\frac{1}{3} \frac{P^{\prime 2}}{M^{\prime 2}}\right)\right]\left(P^{\prime} \overleftrightarrow{\partial}_{P^{\prime}}-P \overleftrightarrow{\partial}_{P}+p^{\prime} \overleftrightarrow{\partial}_{p^{\prime}}\right.} \\
& \left.-p \overleftrightarrow{\partial}_{p}+k^{\prime} \overleftrightarrow{\partial}_{k^{\prime}}-k \overleftrightarrow{\partial}_{k}\right)+\frac{G}{\pi}\left[-2-\frac{2}{3}\left(\frac{P^{2}}{M^{2}}+\frac{P^{\prime 2}}{M^{\prime 2}}\right)\right] \\
& \times\left[M^{\prime}\left(p \overleftrightarrow{\partial}_{p}+k \overleftrightarrow{\partial}_{k}\right)-M\left(p^{\prime} \overleftrightarrow{\partial}_{p^{\prime}}-k^{\prime} \overleftrightarrow{\partial}_{k^{\prime}}\right)\right]
\end{aligned}
$$

\section{Conclusions and remarks}

In this paper, we computed the sub-leading order expansion of the gravitational radiation energy spectrum $d E^{G W} / d \omega$ in the ZFL for a generic multi-body or multi-particle collision. Our result can be applied on both massless and massive fundamental/composite particle scatterings with every spin as well as to bodies such as Black Holes.

As an application, we considered the case of sub-leading soft emission from a gravitational elastic scattering. From the point of view of $\mathrm{BH}$ physics, it can be the case of a test body deflected, but not captured, by a BH.

Surprisingly, we obtain that all sub-leading terms of the energy radiation spectrum, emitted from an elastic collision, exactly cancel each others, in both massless and massive particle collisions. On the other hand, for inelastic $2 \rightarrow 2$ scatterings, we obtain a general and complicated non-zero analytic result which may be applied in the case of GW soft emission from $\mathrm{BH}$ gravitational inelastic scatterings. It may certainly be an attractive possibility to relate our result on searches of BMS gravitational memory effects from GW physics. In this sense, our results may be applied in $\mathrm{BH}$ mergings seen as a gravitational $\mathrm{BH}$ capture inelastic scattering process. On the other hand, violations of universality of our results from loop radiative corrections would be expected from previous analysis as mentioned in the introduction above. 
Another intriguing possibility is to consider footprints of string theory effects on leading and sub-leading gravitational radiation from $\alpha^{\prime}$ corrections and Regge poles that may survive with a polynomial decay in radius, dubbed string memory effect [35].

Finally, the very next step for ZFL program would be to compute sub-sub-leading order on the $d E^{G W} / d \omega$ expansion in $\omega$. In ref. [41] the case of massless particle high energy collisions was considered - having in mind an application to transplanckian scattering regimes. However a more general result is missing yet and it is beyond the purpose of this paper.

\section{A Gravitational elastic scattering I}

In this appendix, we give a detail computation of the differential $B$-factor for the gravitational elastic scattering process with the test particle being massless as discussed in section 4 . In the center of mass frame, the kinematical variables of this process are

$$
\begin{aligned}
& p_{1}=\left(M+\frac{P^{2}}{2 M}, p, k, 0\right), \\
& p_{2}=(P,-p,-k, 0), \\
& p_{3}=\left(-P, p^{\prime},-k^{\prime}, 0\right), \\
& p_{4}=\left(-M-\frac{P^{2}}{2 M},-p^{\prime}, k^{\prime}, 0\right),
\end{aligned}
$$

where $p, p^{\prime}, k, k^{\prime}$ satisfy the realtion

$$
p^{2}+k^{2}=P^{2}=p^{\prime 2}+k^{\prime 2} .
$$

Some properties relating eq. (3.29) are the following

$$
\begin{aligned}
P & =p_{1}+p_{2}=\left(M+P+\frac{P^{2}}{2 M}, \overrightarrow{0}\right), \\
s & =-\left(p_{1}+p_{2}\right)^{2}=\left(M+P+\frac{P^{2}}{2 M}\right)^{2}, \\
\tilde{s}_{i j} & =-\left(M+P+\frac{P^{2}}{2 M}\right)^{2} \frac{1+\vec{v}_{i} \cdot \vec{v}_{j}}{1-\vec{v}_{i} \cdot \vec{v}_{j}}, \\
Q_{i j}^{\mu} & =-\left(M+P+\frac{P^{2}}{2 M}\right)\left(\frac{1+\vec{v}_{i} \cdot \vec{v}_{j}}{1-\vec{v}_{i} \cdot \vec{v}_{j}}, \frac{\vec{v}_{i}+\vec{v}_{j}}{1-\vec{v}_{i} \cdot \vec{v}_{j}}\right),
\end{aligned}
$$

and the velocities are given by

$$
\begin{array}{llll}
\vec{v}_{1}=\frac{1}{M}(p, k, 0), & \vec{v}_{2}=\frac{1}{P}(-p,-k, 0), & \left|\vec{v}_{1}\right|=\frac{P}{M}, & \left|\vec{v}_{2}\right|=\frac{P}{\sqrt{m^{2}+P^{2}}} \rightarrow 1, \\
\vec{v}_{3}=\frac{1}{P}\left(-p^{\prime}, k^{\prime}, 0\right), & \vec{v}_{4}=\frac{1}{M}\left(p^{\prime},-k^{\prime}, 0\right), & \left|\vec{v}_{3}\right|=\frac{P}{\sqrt{m^{2}+P^{2}}} \rightarrow 1, & \left|\vec{v}_{4}\right|=\frac{P}{M} .
\end{array}
$$


Their are six $(i, j)$-pairs we need to take into consideration, namely: $(1,2),(1,3),(1,4)$, $(2,3),(2,4),(3,4)$. After some tedious but straightforward computations, we obtain the contributions from $(1,2)+(3,4)$ :

$$
\begin{aligned}
& \frac{d B_{1}^{(1,2)}}{d \omega}+\frac{d B_{1}^{(2,1)}}{d \omega}+\frac{d B_{1}^{(3,4)}}{d \omega}+\frac{d B_{1}^{(4,3)}}{d \omega} \\
= & \frac{G}{\pi}\left[\ln \left(\frac{M+P+\frac{P^{2}}{2 M}}{M}\right)^{2}+\frac{M}{P} \ln \frac{M-P}{M+P}\right] \frac{M+P}{M-P}\left(P-M-\frac{P^{2}}{2 M}\right) \\
& \times\left(p^{\prime} \overleftrightarrow{\partial}_{p^{\prime}}+k^{\prime} \overleftrightarrow{\partial}_{k^{\prime}}-p \overleftrightarrow{\partial}_{p}-k \overleftrightarrow{\partial}_{k}\right),
\end{aligned}
$$

and from $(1,3)+(2,4)$ :

$$
\begin{aligned}
& \frac{d B_{1}^{(1,3)}}{d \omega}+\frac{d B_{1}^{(3,1)}}{d \omega}+\frac{d B_{1}^{(2,4)}}{d \omega}+\frac{d B_{1}^{(4,2)}}{d \omega} \\
= & \frac{G}{\pi} \frac{P M+\left(p p^{\prime}-k k^{\prime}\right)}{P M-\left(p p^{\prime}-k k^{\prime}\right)}\left[\ln \left(\frac{P\left(M+\frac{P^{2}}{2 M}\right)+p p^{\prime}-k k^{\prime}}{M P}\right)^{2}+\frac{M}{P} \ln \frac{M-P}{M+P}\right] \\
& \times\left\{( P ( M + \frac { P ^ { 2 } } { 2 M } ) + ( p p ^ { \prime } - k k ^ { \prime } ) ) \left[\frac{\left(p-p^{\prime}\right)(P+M)}{P M+\left(p p^{\prime}-k k^{\prime}\right)}\left(\overleftrightarrow{\partial}_{p}+\overleftrightarrow{\partial}_{p^{\prime}}\right)\right.\right. \\
& \left.+\frac{\left(k+k^{\prime}\right)(P+M)}{P M+\left(p p^{\prime}-k k^{\prime}\right)}\left(\overleftrightarrow{\partial}_{k}-\overleftrightarrow{\partial}_{k^{\prime}}\right)\right]+\frac{P\left[P^{2}-\left(M+\frac{P^{2}}{2 M}\right)^{2}\right]}{P\left(M+\frac{P^{2}}{2 M}\right)+p p^{\prime}-k k^{\prime}} \\
& \left.\times\left(p \overleftrightarrow{\partial}_{p^{\prime}}-k \overleftrightarrow{\partial}_{k^{\prime}}-p^{\prime} \overleftrightarrow{\partial}_{p}+k^{\prime} \overleftrightarrow{\partial}_{k}\right)\right\}
\end{aligned}
$$

from $(1,4)$ :

$$
\begin{aligned}
& \frac{d B_{1}^{(1,4)}}{d \omega}+\frac{d B_{1}^{(4,1)}}{d \omega} \\
& =\frac{G}{\pi}\left[\frac{M}{\sqrt{2} \sqrt{P^{2}-\left(p p^{\prime}-k k^{\prime}\right)}} \ln \frac{1+\sqrt{2} \frac{\sqrt{P^{2}-\left(p p^{\prime}-k k^{\prime}\right)}}{M}}{1-\sqrt{2} \frac{\sqrt{P^{2}-\left(p p^{\prime}-k k^{\prime}\right)}}{M}}+2 \frac{M}{P} \ln \frac{M-P}{M+P}\right] M \\
& \times\left(p \overleftrightarrow{\partial}_{p}-p^{\prime} \overleftrightarrow{\partial}_{p^{\prime}}+k \overleftrightarrow{\partial}_{k}-k^{\prime} \overleftrightarrow{\partial}_{k^{\prime}}\right)
\end{aligned}
$$

and finally from $(2,3)$ :

$$
\begin{aligned}
& \frac{d B_{1}^{(2,3)}}{d \omega}+\frac{d B_{1}^{(3,2)}}{d \omega} \\
= & \frac{G}{\pi} \frac{P^{2}-\left(p p^{\prime}-k k^{\prime}\right)}{P^{2}+\left(p p^{\prime}-k k^{\prime}\right)} \ln \left[\frac{P^{2}-\left(p p^{\prime}-k k^{\prime}\right)}{2 P^{2}}\right]^{2}\left[P\left(p+p^{\prime}\right)\left(\overleftrightarrow{\partial}_{p}-\overleftrightarrow{\partial}_{p^{\prime}}\right)-P\left(k^{\prime}-k\right)\left(\overleftrightarrow{\partial}_{k}+\overleftrightarrow{\partial}_{k^{\prime}}\right)\right] .
\end{aligned}
$$


To get a compact and meaningful differential $B$-factor, we do the Taylor series expansion up to the second order at $P / M$. Then the above results are simplified to

$$
\begin{aligned}
& \frac{d B_{1}^{(1,2)}}{d \omega}+\frac{d B_{1}^{(2,1)}}{d \omega}+\frac{d B_{1}^{(3,4)}}{d \omega}+\frac{d B_{1}^{(4,3)}}{d \omega} \sim \frac{G M}{\pi}\left(2-\frac{1}{3} \frac{P^{2}}{M^{2}}\right)\left(p^{\prime} \overleftrightarrow{\partial}_{p^{\prime}}+k^{\prime} \overleftrightarrow{\partial}_{k^{\prime}}-p \overleftrightarrow{\partial}_{p}-k \overleftrightarrow{\partial}_{k}\right), \\
& \frac{d B_{1}^{(1,3)}}{d \omega}+\frac{d B_{1}^{(3,1)}}{d \omega}+\frac{d B_{1}^{(2,4)}}{d \omega}+\frac{d B_{1}^{(4,2)}}{d \omega} \sim \frac{G M}{\pi}\left(-2+\frac{1}{3} \frac{P^{2}}{M^{2}}\right)\left(p \overleftrightarrow{\partial}_{p}-p^{\prime} \overleftrightarrow{\partial}_{p^{\prime}}+k \overleftrightarrow{\partial}_{k}-k^{\prime} \overleftrightarrow{\partial}_{k^{\prime}}\right),
\end{aligned}
$$

and

$$
\begin{aligned}
\frac{d B_{1}^{(1,4)}}{d \omega}+\frac{d B_{1}^{(4,1)}}{d \omega} & =\frac{2 G}{\pi}\left[1+\frac{M}{P} \ln \frac{1-\frac{P}{M}}{1+\frac{P}{M}}\right] M\left(p \overleftrightarrow{\partial}_{p}-p^{\prime} \overleftrightarrow{\partial}_{p^{\prime}}+k \overleftrightarrow{\partial}_{k}-k^{\prime} \overleftrightarrow{\partial}_{k^{\prime}}\right) \\
& \sim \frac{2 G}{\pi}\left[-1-\frac{2}{3} \frac{P^{2}}{M^{2}}\right] M\left(p \overleftrightarrow{\partial}_{p}-p^{\prime} \overleftrightarrow{\partial}_{p^{\prime}}+k \overleftrightarrow{\partial}_{k}-k^{\prime} \overleftrightarrow{\partial}_{k^{\prime}}\right)
\end{aligned}
$$

Summing up the above results, we find that the total differential $B$-factor: $d B_{1} / d \omega=$ $\sum_{i, j}^{i \neq j} d B_{1}^{(i, j)} / d \omega$ has a leading expression as

$$
\begin{aligned}
\frac{d B_{1}}{d \omega} & \sim \frac{G M}{\pi}\left(6+\frac{2}{3} \frac{P^{2}}{M^{2}}\right)\left(p^{\prime} \overleftrightarrow{\partial}_{p^{\prime}}+k^{\prime} \overleftrightarrow{\partial}_{k^{\prime}}-p \overleftrightarrow{\partial}_{p}-k \overleftrightarrow{\partial}_{k}\right) \\
& =\frac{G M}{\pi}\left(6+\frac{2}{3} \frac{P^{2}}{M^{2}}\right)\left(\vec{p}_{f} \overleftrightarrow{\partial}_{\vec{p}_{f}}-\vec{p}_{i} \overleftrightarrow{\partial}_{\vec{p}_{i}}\right)
\end{aligned}
$$

where $\vec{p}_{i}\left(\vec{p}_{f}\right)$ are momenta of initial (final) states and $\overleftrightarrow{\partial} \equiv \overleftarrow{\partial}+\vec{\partial}$

\section{B Gravitational elastic scattering II}

Our goal in this appendix is to generalize the discussion in appendix A to the case in which the test particle has mass $m$. The kinematics is similar to the previous case and reads

$$
\begin{aligned}
& p_{1}=\left(M+\frac{P^{2}}{2 M}, p, k, 0\right), \\
& p_{2}=\left(\sqrt{P^{2}+m^{2}},-p,-k, 0\right), \\
& p_{3}=\left(-\sqrt{P^{2}+m^{2}}, p^{\prime},-k^{\prime}, 0\right), \\
& p_{4}=\left(-M-\frac{P^{2}}{2 M},-p^{\prime}, k^{\prime}, 0\right),
\end{aligned}
$$

with $p^{2}+k^{2}=P^{2}=p^{2}+k^{\prime 2}$.

Again, let us consider the contributions from six pairs separately: first for $(1,2)+(3,4)$, considering that $\tilde{s}_{12}=\tilde{s}_{34}$ and $p_{1} \cdot p_{2}=p_{3} \cdot p_{4}$, we obtain

$$
\begin{aligned}
\frac{d B_{1}^{(1,2)}}{d \omega}+\frac{d B_{1}^{(2,1)}}{d \omega}= & -\frac{G \sqrt{s}}{\pi} \frac{1}{\tilde{s}_{12}}\left[\frac{1}{\beta_{12}} \ln \frac{1+\beta_{12}}{1-\beta_{12}}+\left(\frac{1}{\left|\vec{v}_{1}\right|} \ln \frac{1-\left|\vec{v}_{1}\right|}{1+\left|\vec{v}_{1}\right|}+\frac{1}{\left|\vec{v}_{2}\right|} \ln \frac{1-\left|\vec{v}_{2}\right|}{1+\left|\vec{v}_{2}\right|}\right)\right] \\
& \times\left[\left(p_{1} p_{2}\right) Q_{12}^{\mu}\left(\overleftrightarrow{\partial}_{1}+\overleftrightarrow{\partial}_{2}\right)_{\mu}+\frac{P p_{1}}{p_{1} p_{2}} p_{2}^{2} \overleftrightarrow{\partial}_{2} p_{1}+\frac{P p_{2}}{p_{1} p_{2}} p_{1}^{2} \overleftrightarrow{\partial}_{1} p_{2}\right],
\end{aligned}
$$


and

$$
\begin{aligned}
\frac{d B_{1}^{(3,4)}}{d \omega}+\frac{d B_{1}^{(4,3)}}{d \omega}= & -\frac{G \sqrt{s}}{\pi} \frac{1}{\tilde{s}_{34}}\left[\frac{1}{\beta_{34}} \ln \frac{1+\beta_{34}}{1-\beta_{34}}+\left(\frac{1}{\left|\vec{v}_{3}\right|} \ln \frac{1-\left|\vec{v}_{3}\right|}{1+\left|\vec{v}_{3}\right|}+\frac{1}{\left|\vec{v}_{4}\right|} \ln \frac{1-\left|\vec{v}_{4}\right|}{1+\left|\vec{v}_{4}\right|}\right)\right] \\
& \times\left[\left(p_{3} p_{4}\right) Q_{34}^{\mu}\left(\overleftrightarrow{\partial}_{3}+\overleftrightarrow{\partial}_{4}\right)_{\mu}+\frac{P p_{3}}{p_{3} p_{4}} p_{4}^{2} \overleftrightarrow{\partial}_{4} p_{3}+\frac{P p_{4}}{p_{3} p_{4}} p_{3}^{2} \overleftrightarrow{\partial}_{3} p_{4}\right],
\end{aligned}
$$

where the $Q^{\mu}$ vectors are given by

$$
\begin{aligned}
& Q_{12}^{\mu}=-\frac{M+\frac{P^{2}}{2 M}+\sqrt{P^{2}+m^{2}}}{M \sqrt{P^{2}+m^{2}}+P^{2}}\left(M \sqrt{P^{2}+m^{2}}-P^{2},\left(\sqrt{P^{2}+m^{2}}-M\right)(p, k, 0)\right), \\
& Q_{34}^{\mu}=-\frac{M+\frac{P^{2}}{2 M}+\sqrt{P^{2}+m^{2}}}{M \sqrt{P^{2}+m^{2}}+P^{2}}\left(M \sqrt{P^{2}+m^{2}}-P^{2},\left(\sqrt{P^{2}+m^{2}}-M\right)\left(p^{\prime},-k^{\prime}, 0\right)\right) .
\end{aligned}
$$

Thus we have

$$
\begin{aligned}
& \frac{d B_{1}^{(1,2)}}{d \omega}+\frac{d B_{1}^{(2,1)}}{d \omega}+\frac{d B_{1}^{(3,4)}}{d \omega}+\frac{d B_{1}^{(4,3)}}{d \omega} \\
= & -\frac{G \sqrt{s}}{\pi} \frac{1}{\tilde{s}_{12}}\left[\frac{1}{\beta_{12}} \ln \frac{1+\beta_{12}}{1-\beta_{12}}+\left(\frac{1}{\left|\vec{v}_{1}\right|} \ln \frac{1-\left|\vec{v}_{1}\right|}{1+\left|\vec{v}_{1}\right|}+\frac{1}{\left|\vec{v}_{2}\right|} \ln \frac{1-\left|\vec{v}_{2}\right|}{1+\left|\vec{v}_{2}\right|}\right)\right] \\
& \times\left\{\frac{P p_{1}}{p_{1} p_{2}} m^{2}\left(p \overleftrightarrow{\partial}_{p}+k \overleftrightarrow{\partial}_{k}-p^{\prime} \overleftrightarrow{\partial}_{p^{\prime}}-k^{\prime} \overleftrightarrow{\partial}_{k^{\prime}}\right)+\frac{P p_{2}}{p_{1} p_{2}}\left[P^{2}-\left(M+\frac{P^{2}}{2 M}\right)^{2}\right]\right. \\
& \left.\times\left(p^{\prime} \overleftrightarrow{\partial}_{p^{\prime}}+k^{\prime} \overleftrightarrow{\partial}_{k^{\prime}}-p \overleftrightarrow{\partial}_{p}-k \overleftrightarrow{\partial}_{k}\right)\right\} .
\end{aligned}
$$

The combination: $p^{\overleftrightarrow{\partial}_{p^{\prime}}}+k^{\prime} \overleftrightarrow{\partial}_{k^{\prime}}-p \overleftrightarrow{\partial}_{p}-k \overleftrightarrow{\partial}_{k}$ factorized implies that $\mathcal{M}^{*}[(1,2)+$ $(3,4)] \mathcal{M}=0$ (see eq. $(5.7)$ ).

Then for $(1,3)+(2,4)$, similarly, considering that $\tilde{s}_{13}=\tilde{s}_{24}, p_{1} \cdot p_{3}=p_{2} \cdot p_{4}$, we obtain

$$
\begin{aligned}
& \frac{d B_{1}^{(1,3)}}{d \omega}+\frac{d B_{1}^{(3,1)}}{d \omega}+\frac{d B_{1}^{(2,4)}}{d \omega}+\frac{d B_{1}^{(4,2)}}{d \omega} \\
= & \frac{G \sqrt{s}}{\pi} \frac{1}{\tilde{s}_{13}}\left[\frac{1}{\beta_{13}} \ln \frac{1+\beta_{13}}{1-\beta_{13}}+\left(\frac{1}{\left|\vec{v}_{1}\right|} \ln \frac{1-\left|\vec{v}_{1}\right|}{1+\left|\vec{v}_{1}\right|}+\frac{1}{\left|\vec{v}_{3}\right|} \ln \frac{1-\left|\vec{v}_{3}\right|}{1+\left|\vec{v}_{3}\right|}\right)\right] \\
& \times\left\{-\left(p_{1} p_{3}\right) \frac{\left(M+\frac{P^{2}}{2 M}+\sqrt{P^{2}+m^{2}}\right)\left(\sqrt{P^{2}+m^{2}}+M\right)}{M \sqrt{P^{2}+m^{2}}-\left(k k^{\prime}-p p^{\prime}\right)}\left(p \overleftrightarrow{\partial}_{p}-p^{\prime} \overleftrightarrow{\partial}_{p^{\prime}}+k \overleftrightarrow{\partial}_{k}-k^{\prime} \overleftrightarrow{\partial}_{k^{\prime}}\right.\right. \\
& \left.-p^{\prime} \overleftrightarrow{\partial}_{p}+p \overleftrightarrow{\partial}_{p^{\prime}}+k^{\prime} \overleftrightarrow{\partial}_{k}-k \overleftrightarrow{\partial}_{k^{\prime}}\right)+\frac{P p_{1}}{p_{1} p_{3}} m^{2}\left[p^{\prime} \overleftrightarrow{\partial}_{p}-p \overleftrightarrow{\partial}_{p^{\prime}}+k \overleftrightarrow{\partial}_{k^{\prime}}-k^{\prime} \overleftrightarrow{\partial}_{k}\right] \\
& \left.+\frac{P p_{2}}{p_{1} p_{3}}\left[P^{2}-\left(M+\frac{P^{2}}{2 M}\right)^{2}\right]\left(p \overleftrightarrow{\partial}_{p^{\prime}}-p^{\prime} \overleftrightarrow{\partial}_{p}+k^{\prime} \overleftrightarrow{\partial}_{k}-k \overleftrightarrow{\partial}_{k^{\prime}}\right)\right\} .
\end{aligned}
$$

Let us now note that all the three terms in the above expression contain the combination $p \overleftrightarrow{\partial}_{p^{\prime}}-p^{\prime} \overleftrightarrow{\partial}_{p}+k^{\prime} \overleftrightarrow{\partial}_{k}-k \overleftrightarrow{\partial}_{k^{\prime}}$, which guarantees the cancellation after the application of 
the expression on the amplitudes

$$
\begin{aligned}
& \mathcal{M}^{*}\left[-p^{\prime} \overleftrightarrow{\partial}_{p}+p \overleftrightarrow{\partial}_{p^{\prime}}+k^{\prime} \overleftrightarrow{\partial}_{k}-k \overleftrightarrow{\partial}_{k^{\prime}}\right] \mathcal{M} \\
= & \frac{16 \pi G\left(2 P^{2}(M+P)^{2}-M^{2} m^{2}\right)}{\left(P^{2}+k k^{\prime}-p p^{\prime}\right)^{2}}\left(p^{\prime 2}-p^{2}+k^{\prime 2}-k^{2}\right) \mathcal{M}=0
\end{aligned}
$$

thus $\mathcal{M}^{*}[(1,3)+(2,4)] \mathcal{M}=0$.

Similarly, the final expression for the contribution from $(2,3)$-pair is

$$
\begin{aligned}
& \frac{d B_{1}^{(2,3)}}{d \omega}+\frac{d B_{1}^{(3,2)}}{d \omega} \\
= & \frac{G \sqrt{s}}{\pi} \frac{1}{\tilde{s}_{23}}\left[\frac{1}{\beta_{23}} \ln \frac{1+\beta_{23}}{1-\beta_{23}}+\frac{2}{\left|\vec{v}_{2}\right|} \ln \frac{1-\left|\vec{v}_{2}\right|}{1+\left|\vec{v}_{2}\right|}\right]\left\{-\left(M+\frac{P^{2}}{2 M}+\sqrt{P^{2}+m^{2}}\right)\right. \\
& \times \sqrt{P^{2}+m^{2}}\left(p \overleftrightarrow{\partial}_{p}-p^{\prime} \overleftrightarrow{\partial}_{p^{\prime}}+k \overleftrightarrow{\partial}_{k}-k^{\prime} \overleftrightarrow{\partial}_{k^{\prime}}+p^{\prime} \overleftrightarrow{\partial}_{p}-p \overleftrightarrow{\partial}_{p^{\prime}}-k^{\prime} \overleftrightarrow{\partial}_{k}+k \overleftrightarrow{\partial}_{k^{\prime}}\right) \\
& \left.-\frac{\left(\sqrt{P^{2}+m^{2}}\right)\left(M+\frac{P^{2}}{2 M}+\sqrt{P^{2}+m^{2}}\right)}{\left(P^{2}+m^{2}\right)-\left(p p^{\prime}-k k^{\prime}\right)} m^{2}\left(p \overleftrightarrow{\partial}_{p^{\prime}}-p^{\prime} \overleftrightarrow{\partial}_{p}+k^{\prime} \overleftrightarrow{\partial}_{k}-k \overleftrightarrow{\partial}_{k^{\prime}}\right)\right\}
\end{aligned}
$$

and from (1,4)-pair

$$
\begin{aligned}
& \frac{d B_{1}^{(1,4)}}{d \omega}+\frac{d B_{1}^{(4,1)}}{d \omega} \\
& =\frac{G}{\pi}\left[\frac{\left(M+\frac{P^{2}}{2 M}\right)^{2}-\left(p p^{\prime}-k k^{\prime}\right)}{\sqrt{\left(2 M^{2}+P^{2}+\frac{P^{4}}{4 M^{2}}\right)-\left(p p^{\prime}-k k^{\prime}\right)} \sqrt{\left(P^{2}+\frac{P^{4}}{4 M^{2}}\right)-\left(p p^{\prime}-k k^{\prime}\right)}}\right. \\
& \times \ln \frac{\left(M+\frac{P^{2}}{2 M}\right)^{2}-\left(p p^{\prime}-k k^{\prime}\right)+\sqrt{\left(2 M^{2}+P^{2}+\frac{P^{4}}{4 M^{2}}\right)-\left(p p^{\prime}-k k^{\prime}\right)} \sqrt{\left(P^{2}+\frac{P^{4}}{4 M^{2}}\right)-\left(p p^{\prime}-k k^{\prime}\right)}}{\left(M+\frac{P^{2}}{2 M}\right)^{2}-\left(p p^{\prime}-k k^{\prime}\right)-\sqrt{\left(2 M^{2}+P^{2}+\frac{P^{4}}{4 M^{2}}\right)-\left(p p^{\prime}-k k^{\prime}\right)} \sqrt{\left(P^{2}+\frac{P^{4}}{4 M^{2}}\right)-\left(p p^{\prime}-k k^{\prime}\right)}} \\
& \left.+2 \frac{M}{P} \ln \frac{M-P}{M+P}\right]\left\{\left[\left(M+\frac{P^{2}}{2 M}\right)^{2}-\left(p p^{\prime}-k k^{\prime}\right)\right]\left(\frac{M}{M^{2}-\left(p p^{\prime}-k k^{\prime}\right)}\right)\right. \\
& \times\left(p \overleftrightarrow{\partial}_{p}+k \overleftrightarrow{\partial}_{k}-p^{\prime} \overleftrightarrow{\partial}_{p^{\prime}}-k^{\prime} \overleftrightarrow{\partial}_{k^{\prime}}+k \overleftrightarrow{\partial}_{k^{\prime}}-p \overleftrightarrow{\partial}_{p^{\prime}}+p^{\prime} \overleftrightarrow{\partial}_{p}-k^{\prime} \overleftrightarrow{\partial}_{k}\right) \\
& \left.\left.+\frac{\left(M+\frac{P^{2}}{2 M}\right)\left[P^{2}-\left(M+\frac{P^{2}}{2 M}\right)^{2}\right]}{\left(M+\frac{P^{2}}{2 M}\right)^{2}-\left(p p^{\prime}-k k^{\prime}\right)}\left(k \overleftrightarrow{\partial}_{k^{\prime}}-p \overleftrightarrow{\partial}_{p^{\prime}}+p^{\prime} \overleftrightarrow{\partial}_{p}-k^{\prime} \overleftrightarrow{\partial}_{k}\right)\right]\right\}
\end{aligned}
$$

Once again, these terms will give us a zero result after applying them to the amplitude.

In conclusion, we have generalized $\mathcal{M}^{*} \frac{d B_{1}}{d \omega} \mathcal{M}=0$ to all gravitational elastic scatterings.

\section{C $\frac{d B_{1}^{(i, j)}}{d \omega}$ for the inelastic case}

In this appendix we show the general results (before expansion) of the $d B_{1}^{(i, j)} / d \omega$ factor for the inelastic scattering discussed in section 6. Adopting the kinematics (6.1), one gets from 
eq. (3.29) contributions from six pairs respectively and the results read ${ }^{2}$

$$
\begin{aligned}
& \frac{d B_{1}^{(1,2)}}{d \omega}+\frac{d B_{1}^{(2,1)}}{d \omega}=\frac{G}{\pi}\left[\ln \left(\frac{M+\frac{P^{2}}{2 M}+P}{M}\right)^{2}+\frac{M}{P} \ln \frac{M-P}{M+P}\right]\left[\left(M+\frac{P^{2}}{2 M}+P\right)\right. \\
& \times\left(P \overleftrightarrow{\partial}_{M+\frac{P^{2}}{2 M}}+P \overleftrightarrow{\partial}_{P}\right)+\left(P-M-\frac{P^{2}}{2 M}\right) \frac{M+P}{M-P} \\
& \left.\times\left(P \overleftrightarrow{\partial}_{M+\frac{P}{2 M}}-p \overleftrightarrow{\partial}_{p}-k \overleftrightarrow{\partial}_{k}\right)\right] \\
& \frac{d B_{1}^{(3,4)}}{d \omega}+\frac{d B_{1}^{(4,3)}}{d \omega}=\frac{G}{\pi}\left[\ln \left(\frac{M^{\prime}+P^{\prime}+\frac{P^{\prime 2}}{2 M}}{M^{\prime}}\right)^{2}+\frac{M^{\prime}}{P^{\prime}} \ln \frac{M^{\prime}-P^{\prime}}{M^{\prime}+P^{\prime}}\right]\left[-\left(M^{\prime}+P^{\prime}+\frac{P^{\prime 2}}{2 M^{\prime}}\right)\right. \\
& \times\left(P^{\prime} \overleftrightarrow{\partial}_{P^{\prime}}+P^{\prime} \overleftrightarrow{\partial}_{M^{\prime}+\frac{P^{\prime 2}}{2 M^{\prime}}}\right)-\left(P^{\prime}-M^{\prime}-\frac{P^{\prime 2}}{2 M^{\prime}}\right) \frac{M^{\prime}+P^{\prime}}{M^{\prime}-P^{\prime}} \\
& \left.\times\left(P^{\prime} \overleftrightarrow{\partial}_{M^{\prime}+\frac{P^{\prime 2}}{2 M^{\prime}}}-p^{\prime} \overleftrightarrow{\partial}_{p^{\prime}}-k^{\prime} \overleftrightarrow{\partial}_{k^{\prime}}\right)\right] \\
& \frac{d B_{1}^{(1,3)}}{d \omega}+\frac{d B_{1}^{(3,1)}}{d \omega}=\frac{G}{\pi} \frac{P^{\prime} M+\left(p p^{\prime}-k k^{\prime}\right)}{P^{\prime} M-\left(p p^{\prime}-k k^{\prime}\right)}\left[\ln \left(\frac{P^{\prime}\left(M+\frac{P^{2}}{2 M}\right)+p p^{\prime}-k k^{\prime}}{M P^{\prime}}\right)^{2}\right. \\
& \left.+\frac{M}{P} \ln \frac{M-P}{M+P}\right]\left\{\left[P^{\prime}\left(M+\frac{P^{2}}{2 M}\right)+\left(p p^{\prime}-k k^{\prime}\right)\left(\frac{P^{\prime} M-\left(p p^{\prime}-k k^{\prime}\right)}{P^{\prime} M+\left(p p^{\prime}-k k^{\prime}\right)}\right.\right.\right. \\
& \times\left(\overleftrightarrow{\partial}_{M+\frac{P^{2}}{2 M}}-\overleftrightarrow{\partial}_{P^{\prime}}\right)+\frac{p P^{\prime}-p^{\prime} M}{P^{\prime} M+\left(p p^{\prime}-k k^{\prime}\right)}\left(\overleftrightarrow{\partial}_{p}+\overleftrightarrow{\partial}_{p^{\prime}}\right) \\
& \left.+\frac{k^{\prime} M+k P^{\prime}}{P^{\prime} M+\left(p p^{\prime}-k k^{\prime}\right)}\left(\overleftrightarrow{\partial}_{k}-\overleftrightarrow{\partial}_{k^{\prime}}\right)\right]-\frac{P^{\prime}\left[P^{2}-\left(M+\frac{P^{2}}{2 M}\right)^{2}\right]}{P^{\prime}\left(M+\frac{P^{2}}{2 M}\right)+p p^{\prime}-k k^{\prime}} \\
& \left.\times\left(-P^{\prime} \overleftrightarrow{\partial}_{M+\frac{P 2}{2 M}}+p^{\prime} \overleftrightarrow{\partial}_{p}-k^{\prime} \overleftrightarrow{\partial}_{k}\right)\right\} \\
& \frac{d B_{1}^{(2,4)}}{d \omega}+\frac{d B_{1}^{(4,2)}}{d \omega}=\frac{G}{\pi} \frac{P M^{\prime}+\left(p p^{\prime}-k k^{\prime}\right)}{P M^{\prime}-\left(p p^{\prime}-k k^{\prime}\right)}\left[\ln \left(\frac{P\left(M^{\prime}+\frac{P^{\prime 2}}{2 M^{\prime}}\right)+p p^{\prime}-k k^{\prime}}{M^{\prime} P}\right)^{2}\right. \\
& \left.+\frac{M^{\prime}}{P^{\prime}} \ln \frac{M^{\prime}-P^{\prime}}{M^{\prime}+P^{\prime}}\right]\left\{[ P ( M ^ { \prime } + \frac { P ^ { \prime 2 } } { 2 M ^ { \prime } } ) + ( p p ^ { \prime } - k k ^ { \prime } ) ] \left[\frac{P M^{\prime}-\left(p p^{\prime}-k k^{\prime}\right)}{P M^{\prime}+\left(p p^{\prime}-k k^{\prime}\right)}\right.\right. \\
& \times\left(\overleftrightarrow{\partial}_{P}-\overleftrightarrow{\partial}_{M^{\prime}+\frac{P^{\prime 2}}{2 M^{\prime}}}\right)-\frac{p^{\prime} P-p M^{\prime}}{P M^{\prime}+\left(p p^{\prime}-k k^{\prime}\right)}\left(\overleftrightarrow{\partial}_{p}+\overleftrightarrow{\partial}_{p^{\prime}}\right) \\
& \left.+\frac{\left(k M^{\prime}+k^{\prime} P\right)}{P M^{\prime}+\left(p p^{\prime}-k k^{\prime}\right)}\left(\overleftrightarrow{\partial}_{k}-\overleftrightarrow{\partial}_{k^{\prime}}\right)\right]+\frac{P\left[P^{\prime 2}-\left(M^{\prime}+\frac{P^{\prime 2}}{2 M^{\prime}}\right)^{2}\right]}{P\left(M^{\prime}+\frac{P^{\prime 2}}{2 M^{\prime}}\right)+p p^{\prime}-k k^{\prime}} \\
& \left.\times\left(-P \overleftrightarrow{\partial}_{M^{\prime}+\frac{P^{\prime 2}}{2 M^{\prime}}}+p \overleftrightarrow{\partial}_{p^{\prime}}-k \overleftrightarrow{\partial}_{k^{\prime}}\right)\right\}
\end{aligned}
$$

\footnotetext{
${ }^{2}$ The computation for the inelastic case is similar to those in appendix A and B (although much more complicated), thus to avoid repeatability we only show the results in this appendix.
} 


$$
\begin{aligned}
& \frac{d B_{1}^{(1,4)}}{d \omega}+\frac{d B_{1}^{(4,1)}}{d \omega} \\
& =\frac{G}{\pi} \frac{M M^{\prime}-\left(p p^{\prime}-k k^{\prime}\right)}{M M^{\prime}+\left(p p^{\prime}-k k^{\prime}\right)}\left[\frac{\left(M+\frac{P^{2}}{2 M}\right)\left(M^{\prime}+\frac{P^{\prime 2}}{2 M^{\prime}}\right)-\left(p p^{\prime}-k k^{\prime}\right)}{\sqrt{\left[\left(M+\frac{P^{2}}{2 M}\right)\left(M^{\prime}+\frac{P^{\prime 2}}{2 M^{\prime}}\right)-\left(p p^{\prime}-k k^{\prime}\right)\right]^{2}-M^{2} M^{\prime 2}}}\right. \\
& \times \ln \frac{\left(M+\frac{P^{2}}{2 M}\right)\left(M^{\prime}+\frac{P^{\prime 2}}{2 M^{\prime}}\right)-\left(p p^{\prime}-k k^{\prime}\right)+\sqrt{\left[\left(M+\frac{P^{2}}{2 M}\right)\left(M^{\prime}+\frac{P^{\prime 2}}{2 M^{\prime}}\right)-\left(p p^{\prime}-k k^{\prime}\right)\right]^{2}-M^{2} M^{\prime 2}}}{\left(M+\frac{P^{2}}{2 M}\right)\left(M^{\prime}+\frac{P^{\prime 2}}{2 M^{\prime}}\right)-\left(p p^{\prime}-k k^{\prime}\right)-\sqrt{\left[\left(M+\frac{P^{2}}{2 M}\right)\left(M^{\prime}+\frac{P^{\prime 2}}{2 M^{\prime}}\right)-\left(p p^{\prime}-k k^{\prime}\right)\right]^{2}-M^{2} M^{\prime 2}}} \\
& \left.+\frac{M}{P} \ln \frac{M-P}{M+P}+\frac{M^{\prime}}{P^{\prime}} \ln \frac{M^{\prime}-P^{\prime}}{M^{\prime}+P^{\prime}}\right]\left\{\left[\left(M+\frac{P^{2}}{2 M}\right)\left(M^{\prime}+\frac{P^{\prime 2}}{2 M^{\prime}}\right)-\left(p p^{\prime}-k k^{\prime}\right)\right]\right. \\
& \times\left[\frac{M M^{\prime}+\left(p p^{\prime}-k k^{\prime}\right)}{M M^{\prime}-\left(p p^{\prime}-k k^{\prime}\right)}\left(\overleftrightarrow{\partial}_{M+\frac{p^{2}}{2 M}}-\overleftrightarrow{\partial}_{M^{\prime}+\frac{P^{\prime 2}}{2 M^{\prime}}}\right)+\frac{p M^{\prime}+p^{\prime} M}{M M^{\prime}-\left(p p^{\prime}-k k^{\prime}\right)}\left(\overleftrightarrow{\partial}_{p}-\overleftrightarrow{\partial}_{p^{\prime}}\right)\right. \\
& \left.+\frac{k M^{\prime}-k^{\prime} M}{M M^{\prime}-\left(p p^{\prime}-k k^{\prime}\right)}\left(\overleftrightarrow{\partial}_{k}+\overleftrightarrow{\partial}_{k^{\prime}}\right)\right]-\frac{\left(M+\frac{P^{2}}{2 M}\right)\left[P^{\prime 2}-\left(M^{\prime}+\frac{P^{\prime 2}}{2 M^{\prime}}\right)^{2}\right]}{\left(M+\frac{P^{2}}{2 M}\right)\left(M^{\prime}+\frac{P^{\prime 2}}{2 M^{\prime}}\right)-\left(p p^{\prime}-k k^{\prime}\right)} \\
& \times\left[\left(M+\frac{P^{2}}{2 M}\right) \overleftrightarrow{\partial}_{M^{\prime}+\frac{P^{\prime 2}}{2 M^{\prime}}}+p \overleftrightarrow{\partial}_{p^{\prime}}-k \overleftrightarrow{\partial}_{k^{\prime}}\right]+\frac{\left(M^{\prime}+\frac{P^{\prime 2}}{2 M^{\prime}}\right)\left[P^{2}-\left(M+\frac{P^{2}}{2 M}\right)^{2}\right]}{\left(M+\frac{P^{2}}{2 M}\right)\left(M^{\prime}+\frac{P^{\prime 2}}{2 M^{\prime}}\right)-\left(p p^{\prime}-k k^{\prime}\right)} \\
& \left.\times\left[\left(M^{\prime}+\frac{P^{\prime 2}}{2 M^{\prime}}\right) \overleftrightarrow{\partial}_{M+\frac{P^{2}}{2 M}}+p^{\prime} \overleftrightarrow{\partial}_{p}-k^{\prime} \overleftrightarrow{\partial}_{k}\right]\right\} \\
& \frac{d B_{1}^{(2,3)}}{d \omega}+\frac{d B_{1}^{(3,2)}}{d \omega} \\
& =\frac{2 G}{\pi} \frac{P P^{\prime}-\left(p p^{\prime}-k k^{\prime}\right)}{P P^{\prime}+\left(p p^{\prime}-k k^{\prime}\right)} \ln \left[\frac{P P^{\prime}-\left(p p^{\prime}-k k^{\prime}\right)}{2 P P^{\prime}}\right]\left\{\left[P P^{\prime}+\left(p p^{\prime}-k k^{\prime}\right)\right]\left(\overleftrightarrow{\partial}_{P}-\overleftrightarrow{\partial}_{P^{\prime}}\right)\right. \\
& \left.+\left(p P^{\prime}+p^{\prime} P\right)\left(\overleftrightarrow{\partial}_{p}-\overleftrightarrow{\partial}_{p^{\prime}}\right)+\left(k P^{\prime}-k^{\prime} P\right)\left(\overleftrightarrow{\partial}_{k}+\overleftrightarrow{\partial}_{k^{\prime}}\right)\right\}
\end{aligned}
$$

\section{Acknowledgments}

AA would like to thank Massimo Bianchi for interesting and useful discussions and remarks on this subject. Our work is supported by the Talent Scientific Research Program of College of Physics, Sichuan University, Grant No.1082204112427 \& the Fostering Program in Disciplines Possessing Novel Features for Natural Science of Sichuan University, Grant No. 2020SCUNL209 \& 1000 Talent program of Sichuan province 2021.

Open Access. This article is distributed under the terms of the Creative Commons Attribution License (CC-BY 4.0), which permits any use, distribution and reproduction in any medium, provided the original author(s) and source are credited. 


\section{References}

[1] L. Smarr, Gravitational radiation from distant encounters and from headon collisions of black holes: the zero frequency limit, Phys. Rev. D 15 (1977) 2069 [InSPIRE].

[2] S. Weinberg, Infrared photons and gravitons, Phys. Rev. 140 (1965) B156.

[3] M. Gell-Mann and M.L. Goldberger, Scattering of low-energy photons by particles of spin 1/2, Phys. Rev. 96 (1954) 1433 [inSPIRE].

[4] F.E. Low, Bremsstrahlung of very low-energy quanta in elementary particle collisions, Phys. Rev. 110 (1958) 974 [INSPIRE].

[5] T.H. Burnett and N.M. Kroll, Extension of the low soft photon theorem, Phys. Rev. Lett. 20 (1968) 86 [INSPIRE].

[6] D.J. Gross and R. Jackiw, Low-energy theorem for graviton scattering, Phys. Rev. 166 (1968) 1287 [INSPIRE].

[7] R. Jackiw, Low-energy theorems for massless bosons: photons and gravitons, Phys. Rev. 168 (1968) 1623 [INSPIRE].

[8] P.P. Kulish and L.D. Faddeev, Asymptotic conditions and infrared divergences in quantum electrodynamics, Theor. Math. Phys. 4 (1970) 745 [InSPIRE].

[9] J.F. Donoghue and T. Torma, Infrared behavior of graviton-graviton scattering, Phys. Rev. D 60 (1999) 024003 [hep-th/9901156] [INSPIRE].

[10] C.D. White, Factorization properties of soft graviton amplitudes, JHEP 05 (2011) 060 [arXiv: 1103.2981] [INSPIRE].

[11] E. Casali, Soft sub-leading divergences in Yang-Mills amplitudes, JHEP 08 (2014) 077 [arXiv:1404.5551] [INSPIRE].

[12] B.U.W. Schwab and A. Volovich, Subleading soft theorem in arbitrary dimensions from scattering equations, Phys. Rev. Lett. 113 (2014) 101601 [arXiv: 1404.7749] [INSPIRE].

[13] N. Afkhami-Jeddi, Soft graviton theorem in arbitrary dimensions, arXiv:1405.3533 [INSPIRE].

[14] M. Bianchi, S. He, Y.-t. Huang and C. Wen, More on soft theorems: Trees, loops, and strings, Phys. Rev. D 92 (2015) 065022 [arXiv:1406.5155] [INSPIRE].

[15] Z. Bern, S. Davies, P. Di Vecchia and J. Nohle, Low-energy behavior of gluons and gravitons from gauge invariance, Phys. Rev. D 90 (2014) 084035 [arXiv:1406.6987] [INSPIRE].

[16] P. Di Vecchia, R. Marotta and M. Mojaza, Soft theorem for the graviton, dilaton and the Kalb-Ramond field in the bosonic string, JHEP 05 (2015) 137 [arXiv:1502.05258] [INSPIRE].

[17] P. Di Vecchia, R. Marotta, M. Mojaza and J. Nohle, New soft theorems for the gravity dilaton and the Nambu-Goldstone dilaton at subsubleading order, Phys. Rev. D 93 (2016) 085015 [arXiv: 1512.03316] [INSPIRE].

[18] M. Bianchi, A.L. Guerrieri, Y.-t. Huang, C.-J. Lee and C. Wen, Exploring soft constraints on effective actions, JHEP 10 (2016) 036 [arXiv:1605. 08697] [INSPIRE].

[19] M. Bianchi and A.L. Guerrieri, On the soft limit of closed string amplitudes with massive states, Nucl. Phys. B 905 (2016) 188 [arXiv:1512.00803] [InSPIRE].

[20] M. Bianchi and A.L. Guerrieri, On the soft limit of tree-level string amplitudes, in 14th Marcel Grossmann Meeting on Recent Developments in Theoretical and Experimental General Relativity, Astrophysics, and Relativistic Field Theories, vol. 4, (2017), pp. 4157-4163, DOI [arXiv: 1601.03457] [INSPIRE]. 
[21] A.L. Guerrieri, Y.-t. Huang, Z. Li and C. Wen, On the exactness of soft theorems, JHEP 12 (2017) 052 [arXiv: 1705.10078] [INSPIRE].

[22] Z. Bern, S. Davies and J. Nohle, On loop corrections to subleading soft behavior of gluons and gravitons, Phys. Rev. D 90 (2014) 085015 [arXiv:1405.1015] [inSPIRE].

[23] A. Sen, Soft theorems in superstring theory, JHEP 06 (2017) 113 [arXiv:1702.03934] [INSPIRE].

[24] A. Sen, Subleading soft graviton theorem for loop amplitudes, JHEP 11 (2017) 123 [arXiv: 1703.00024] [INSPIRE].

[25] A. Laddha and A. Sen, Sub-subleading soft graviton theorem in generic theories of quantum gravity, JHEP 10 (2017) 065 [arXiv:1706.00759] [INSPIRE].

[26] S. Chakrabarti, S.P. Kashyap, B. Sahoo, A. Sen and M. Verma, Testing subleading multiple soft graviton theorem for CHY prescription, JHEP 01 (2018) 090 [arXiv:1709. 07883] [INSPIRE].

[27] F. Cachazo and A. Strominger, Evidence for a new soft graviton theorem, arXiv:1404.4091 [INSPIRE].

[28] R. Britto, F. Cachazo and B. Feng, New recursion relations for tree amplitudes of gluons, Nucl. Phys. B 715 (2005) 499 [hep-th/0412308] [INSPIRE].

[29] R. Britto, F. Cachazo, B. Feng and E. Witten, Direct proof of tree-level recursion relation in Yang-Mills theory, Phys. Rev. Lett. 94 (2005) 181602 [hep-th/0501052] [INSPIRE].

[30] A. Strominger, On BMS invariance of gravitational scattering, JHEP 07 (2014) 152 [arXiv: 1312.2229] [INSPIRE].

[31] S.W. Hawking, M.J. Perry and A. Strominger, Superrotation charge and supertranslation hair on black holes, JHEP 05 (2017) 161 [arXiv: 1611.09175] [INSPIRE].

[32] A. Strominger and A. Zhiboedov, BMS supertranslations and soft theorems, JHEP 01 (2016) 086 [arXiv: 1411.5745] [INSPIRE].

[33] LIGO SCIENTIFIC and VIRGO collaborations, GW170817: observation of gravitational waves from a binary neutron star inspiral, Phys. Rev. Lett. 119 (2017) 161101 [arXiv:1710.05832] [INSPIRE].

[34] M.D. Johnson et al., Universal interferometric signatures of a black hole's photon ring, Sci. Adv. 6 (2020) eaaz1310 [arXiv:1907.04329] [INSPIRE].

[35] A. Addazi, M. Bianchi, M. Firrotta and A. Marcianò, String memories... lost and regained, Nucl. Phys. B 965 (2021) 115356 [arXiv:2008.02206] [INSPIRE].

[36] A. Aldi, M. Bianchi and M. Firrotta, String memories... openly retold, Phys. Lett. B 813 (2021) 136037 [arXiv: 2010.04082] [INSPIRE].

[37] A. Aldi, M. Bianchi and M. Firrotta, Spinning-off stringy electro-magnetic memories, arXiv:2101.07054 [INSPIRE].

[38] T. Ikeda, M. Bianchi, D. Consoli, A. Grillo, J.F. Morales, P. Pani et al., Black-hole microstate spectroscopy: Ringdown, quasinormal modes, and echoes, Phys. Rev. D 104 (2021) 066021 [arXiv:2103.10960] [INSPIRE].

[39] A. Laddha and A. Sen, Gravity waves from soft theorem in general dimensions, JHEP 09 (2018) 105 [arXiv: 1801.07719] [INSPIRE].

[40] B. Sahoo and A. Sen, Classical and quantum results on logarithmic terms in the soft theorem in four dimensions, JHEP 02 (2019) 086 [arXiv: 1808.03288] [INSPIRE]. 
[41] A. Addazi, M. Bianchi and G. Veneziano, Soft gravitational radiation from ultra-relativistic collisions at sub- and sub-sub-leading order, JHEP 05 (2019) 050 [arXiv:1901.10986] [INSPIRE].

[42] B. Sahoo and A. Sen, Classical soft graviton theorem rewritten, arXiv:2105.08739 [INSPIRE].

[43] M. Ciafaloni, D. Colferai and G. Veneziano, Infrared features of gravitational scattering and radiation in the eikonal approach, Phys. Rev. D 99 (2019) 066008 [arXiv:1812.08137] [INSPIRE].

[44] D. Amati, M. Ciafaloni and G. Veneziano, Superstring collisions at planckian energies, Phys. Lett. B 197 (1987) 81 [inSPIRE].

[45] I.J. Muzinich and M. Soldate, High-energy unitarity of gravitation and strings, Phys. Rev. D 37 (1988) 359 [INSPIRE].

[46] G. 't Hooft, Graviton dominance in ultra-high-energy scattering, Phys. Lett. B 198 (1987) 61 [INSPIRE].

[47] D. Amati, M. Ciafaloni and G. Veneziano, Classical and quantum gravity effects from planckian enrgy superstring collisions, Int. J. Mod. Phys. A 3 (1988) 1615 [INSPIRE].

[48] D.J. Gross and P.F. Mende, The high-energy behavior of string scattering amplitudes, Phys. Lett. B 197 (1987) 129 [INSPIRE].

[49] D.J. Gross and P.F. Mende, String theory beyond the Planck scale, Nucl. Phys. B 303 (1988) 407 [INSPIRE].

[50] P.F. Mende and H. Ooguri, Borel summation of string theory for Planck scale scattering, Nucl. Phys. B 339 (1990) 641 [INSPIRE].

[51] D. Amati, M. Ciafaloni and G. Veneziano, Higher order gravitational deflection and soft Bremsstrahlung in planckian energy superstring collisions, Nucl. Phys. B 347 (1990) 550 [INSPIRE].

[52] D. Amati, M. Ciafaloni and G. Veneziano, Towards an S-matrix description of gravitational collapse, JHEP 02 (2008) 049 [arXiv: 0712.1209] [INSPIRE].

[53] G. Veneziano, String-theoretic unitary S-matrix at the threshold of black-hole production, JHEP 11 (2004) 001 [hep-th/0410166] [INSPIRE].

[54] A. Gruzinov and G. Veneziano, Gravitational radiation from massless particle collisions, Class. Quant. Grav. 33 (2016) 125012 [arXiv:1409.4555] [INSPIRE].

[55] M. Ciafaloni, D. Colferai, F. Coradeschi and G. Veneziano, Unified limiting form of graviton radiation at extreme energies, Phys. Rev. D 93 (2016) 044052 [arXiv:1512.00281] [INSPIRE].

[56] M. Ciafaloni, D. Colferai and G. Veneziano, Emerging Hawking-like radiation from gravitational Bremsstrahlung beyond the Planck scale, Phys. Rev. Lett. 115 (2015) 171301 [arXiv: 1505.06619] [INSPIRE].

[57] M. Ciafaloni and D. Colferai, Radiation enhancement and temperature in the collapse regime of gravitational scattering, Phys. Rev. D 95 (2017) 086003 [arXiv:1612.06923] [INSPIRE].

[58] D.M. Eardley and S.B. Giddings, Classical black hole production in high-energy collisions, Phys. Rev. D 66 (2002) 044011 [gr-qc/0201034] [INSPIRE].

[59] E. Kohlprath and G. Veneziano, Black holes from high-energy beam-beam collisions, JHEP 06 (2002) 057 [gr-qc/0203093] [INSPIRE].

[60] H. Yoshino and Y. Nambu, Black ring formation in particle systems, Phys. Rev. D 70 (2004) 084036 [gr-qc/0404109] [INSPIRE]. 
[61] S.B. Giddings and V.S. Rychkov, Black holes from colliding wavepackets, Phys. Rev. D 70 (2004) 104026 [hep-th/0409131] [INSPIRE].

[62] S.W. Hawking, Particle creation by black holes, Commun. Math. Phys. 43 (1975) 199 [Erratum ibid. 46 (1976) 206] [INSPIRE].

[63] S.B. Giddings, Locality in quantum gravity and string theory, Phys. Rev. D 74 (2006) 106006 [hep-th/0604072] [INSPIRE].

[64] D. Amati, M. Ciafaloni and G. Veneziano, Can space-time be probed below the string size?, Phys. Lett. B 216 (1989) 41 [INSPIRE].

[65] G. D'Appollonio, P. Di Vecchia, R. Russo and G. Veneziano, Regge behavior saves string theory from causality violations, JHEP 05 (2015) 144 [arXiv:1502.01254] [INSPIRE].

[66] A. Addazi, M. Bianchi and G. Veneziano, Glimpses of black hole formation/evaporation in highly inelastic, ultra-planckian string collisions, JHEP 02 (2017) 111 [arXiv:1611.03643] [INSPIRE].

[67] N.E.J. Bjerrum-Bohr, P.H. Damgaard, G. Festuccia, L. Planté and P. Vanhove, General relativity from scattering amplitudes, Phys. Rev. Lett. 121 (2018) 171601 [arXiv:1806.04920] [INSPIRE]. 